\title{
Time and Space in Digital Game Storytelling
}

\author{
Huaxin Wei, Jim Bizzocchi, and Tom Calvert \\ School of Interactive Arts and Technology, Simon Fraser University, 250-13450 102 Avenue, Surrey, BC, Canada V3T 0A3 \\ Correspondence should be addressed to Huaxin Wei, huaxinw@sfu.ca
}

Received 1 May 2010; Accepted 7 November 2010

Academic Editor: Abdennour El Rhalibi

Copyright (C) 2010 Huaxin Wei et al. This is an open access article distributed under the Creative Commons Attribution License, which permits unrestricted use, distribution, and reproduction in any medium, provided the original work is properly cited.

\begin{abstract}
The design and representation of time and space are important in any narrative form. Not surprisingly there is an extensive literature on specific considerations of space or time in game design. However, there is less attention to more systematic analyses that examine both of these key factors - including their dynamic interrelationship within game storytelling. This paper adapts critical frameworks of narrative space and narrative time drawn from other media and demonstrates their application in the understanding of game narratives. In order to do this we incorporate fundamental concepts from the field of game studies to build a game-specific framework for analyzing the design of narrative time and narrative space. The paper applies this framework against a case analysis in order to demonstrate its operation and utility. This process grounds the understanding of game narrative space and narrative time in broader traditions of narrative discourse and analysis.
\end{abstract}

\section{Introduction}

Thanks to the rapid innovation in computational technologies, interactive media are becoming more and more dominant in communication and entertainment. As technical limitations disappear, artists and designers feel more at ease using interactive media as their vehicles for expression. Storytelling has found a new home in interactive media implemented in multimedia, websites, hypertexts, interactive fictions and films, as well as digital games. Among these forms, digital games have undoubtedly received most attention and popularity, and have become the most successful application of interactive narrative. Despite the debate that has centred on the potential conflict between interactivity and the experience of narrative, the past decade has seen increasing effort applied to creating meaningful and engaging stories in interactive media, especially digital games. This effort has quietly addressed the issue and has significantly advanced the power of storytelling to enhance the audience's experience. This can be seen in such successful commercial titles as the Assassin's Creed series, the Prince of Persia series, and the Fable series, as well as Mateas and Stern's noncommercial title Façade. Wolf has pointed out that "[a]s the video game's use of space and time grew more complex and graphics grew more representational, the medium became increasingly narrative based" [1]. Indeed, in the construction of any kind of narrative, time and space play a crucial role, not only in the process of narrative authoring, but also in the process of narrative comprehension. Between the two, narrative comprehension is especially linked to a player's experience of a game. As Bridgeman put it: " $[\mathrm{t}]$ o read a narrative is to engage with an alternative world that has its own temporal and spatial structures" [2], the temporal and spatial design of a game, therefore, determines the ways that players engage with the game. Critical analyses of narrative implications of the construction of time and space in games can facilitate an understanding of the mechanism of interactive storytelling, for today's narrativebased games demonstrate the most sophisticated design and technology for digital interactive storytelling systems. This understanding will support more effective approaches in future narrative design and provide an objective basis for analyzing interactive narratives.

Time and space are equally important for storytelling in digital games. Through a look at past game studies, we can see contributions to both aspects and arguments promoting both. Game space has probably been explicitly recognized by more researchers and designers in the field. It is obvious that in order to design a game it is necessary to design a space. This strong recognition can be summarized by 
Aarseth's claim: "The defining element in computer games is spatiality. Computer games are essentially concerned with spatial representation and negotiation; therefore the classification of a computer game can be based on how it represents or, perhaps, implements space" [3]. Along this line, there exist various classifications of game space. For example, Wolf identifies eleven spatial structures based on the dichotomy of on-screen and off-screen space used by film theory [4]; Boron takes a historical view and describes fifteen types of game space [5]; Jenkins directly connects game space to narrative experience and suggests four ways in which the structuring of game space can facilitate narrative experience. As Jenkins states: "spatial stories can evoke preexisting narrative associations; they can provide a staging ground where narrative events are enacted; they may embed narrative information within their mise-en-scene; or they provide resources for emergent narratives" [6]. Using a player's experiential point of view to examine game time, Nistche concludes that the mapping of game time onto the game world can only be done with spatial reference thanks to the continuity of space [7]. The implication here is that time in games can be stopped, reversed, or altered in other ways, which can cause trouble when we try to denote a specific time point in a game; spatial reference is relatively more stable. Incorporating architectural approaches into the study of video game space functionality, Nitsche identifies such spatial structures as tracks/rails, labyrinths/mazes, and arenas. Similar to Jenkins, he observes that evocative narrative elements can be organized or placed according to the spatial structure; the player's experience is hence driven by the spatial structure.

Game time is just as important as game space in the understanding of game design and game experience. Digital games are a temporal medium-where players drive the gameplay forward to completion while the game narrative unfolds over time. Such frequently discussed topics in game narrative as repetition, pacing, dramatic arc, and closure, as well as such mechanics as deceleration and the ticking clock [8], all fall into the realm of temporality. Games do inherit certain temporal design conventions from older media like film. However, due to the dynamic nature of ludic gameplay, augmented by the power of computation, time in digital games also has unique characteristics that facilitate or mediate both the gameplay and the associated narrative.

Time and space can be seen as separate-but not unconnected-aspects of game design and experience. As Arsenault and Perron argue, "[w]e should not forget that the temporal dimension of gameplay prevails on its spatial characterization," since gameplay occurs through a series of interactions that take place in patterns of reflexive and cyclic progression [9]. In our construction of a story world, time and space are two aspects that complement and reference each other. While the literature mentioned above tackles time or space from various angles, there is no systematic account of how time and space are structured in game narratives. In previous work, spatial analyses have not gone much beyond the classification of topological spatial structures. Temporal analyses, similarly, have been limited to identifying a few typologies of time schemes or frames, such as Juul's dual structure (play time and fictional time) [10] and Zagal and Mateas's four frames (real-world time, gameworld time, coordination time, fictive time) [11].

Compared with this largely undefined area of game narrative, time and space have been studied extensively in broader narrative theory. Both have mature descriptive frameworks that have been tested and refined in the long practice of narrative analysis. A mindful adaptation of narrative theory for game studies will help us understand game storytelling better and help build up context-specific principles for game narrative design. As Ryan concludes, " $[t]$ he inability of literary narratology to account for the experience of games does not mean that we should throw away the concept of narrative in ludology; it rather means that we need to expand the catalog of narrative modalities beyond the diegetic and the dramatic, by adding a phenomenological category tailor-made for games" [12]. To do this, we pay special attention to the interactive nature of game storytelling. Our starting point is to examine how time and space are structured in narrative in general, then incorporate insights from the field of game design and game studies, and finally reach a game-specific description of the structural aspects of time and space in game narratives. After an overview of the foundational concepts we use for game narrative analysis, we will delineate in detail how these aspects characterize narrative time and space in games, and how time and space converge in the construction of the plot. The analysis is illustrated and supported with relevant game examples. In our discussion, despite the focus on game storytelling, we will always place the concepts and approaches in the bigger context of interactive storytelling systems. Occasionally we will draw in other interactive narrative examples in an effort to demonstrate the applicability of our game-specific framework to general interactive storytelling systems.

\section{Analyzing Time and Space in Game Narrative}

In narrative theory, there has been more discussion on temporality than spatiality. Stories are commonly framed as sequences of events (hence temporality is the ruling aspect) and space as a mere static description interspersed into narration. This opinion about time/space opposition, however, has been challenged by many who consider that time and space are closely bound together in narrative. Bakhtin was the most famous scholar who challenged the time/space opposition and proposed the concept of chronotope, i.e., the space-time complex, to refer to the connectedness of temporal and spatial relationships in narrative [13]. Zoran is another scholar who criticizes the opposition [14]. Taking a cognitive perspective, Herman considers narrative comprehension "a process of (re)constructing storyworlds on the basis of textual cues and in the inferences that they make possible" and promotes the notion of storyworld as an integrated view of narrative, with time and space being two important and complementary aspects [15]. The cognitive process of narrative comprehension is analogous to what a player goes through during gameplay. Through this process, as Jenkins observes, players, like film spectators, form their 
"mental maps of the narrative action and the story space" and act upon those mental maps "to test them against the game world itself" [6]. Nitsche also argues that players understand the space and movement in games by way of narrative comprehension. His study of video games views narrative as "a form of understanding of the events a player causes, triggers, and encounters inside a video game space" [16]. A focus on audiences' mental process will broaden the scope of narrative defined by the traditional mimesis (story as acted) and diegesis (story as told) and thus potentially gives room to develop a user-centric approach to games as suggested by Ryan quoted earlier, which addresses story as generated from a phenomenological perspective [12]. In this work, we adapt Herman's notion of storyworld to refer to the world a digital game creates through textual, visual, auditory, and haptic cues, where players draw inferences and drive the game to completion through a series of events. A game's storyworld will not be complete without players' mental (re)construction activities that help bridge the gaps left in the computer-generated virtual world. Time and space, consequently, are two important aspects in the reconstruction of a storyworld.

\subsection{Story Time, Operational Time, and Fuzzy Temporality.} In narrative theory, most of the approaches to time depart from the basic distinction of two temporalities in narrative: story time and discourse time. To put it simply, story is about what is depicted in a narrative and discourse is about how it is told. As story is the basic sequence of events, story time is considered the chronological time when the events happened. Discourse time, on the other hand, can be understood differently under different contexts. As Bridgeman points out, in oral narratives, discourse time can be the time of telling; whereas in written narratives, since we cannot access the act of writing, discourse time can be generally referred to as the time of reading [2]. In some narratives, story and discourse times are simply matched, but in many narratives, these two can be very different. The temporal relationships between the two schemes thus produce many interesting narrative effects. These relationships are best classified by Genette as order, duration, and frequency [17]. These three categories have been very popular and adopted by numerous works in narrative analysis; however, they are all based on the assumption that the relation between story time and discourse time can be determined. Studies of many recent narrative texts have seen the indeterminacy of temporality. Herman uses "fuzzy temporality" to describe a subtype of temporal relations that involve "temporal sequencing that is strategically inexact, making it difficult or even impossible to assign narrated events a fixed or even fixable position along a timeline in the storyworld" [15]. He uses "polychrony" to cover all types of narration with fuzzy temporality, including both temporal indefiniteness (i.e., events are partially ordered) and temporal multiplicity (i.e., events are ordered in multiple sequences). "Polychrony" is related to the notion of "achrony" but with bigger scope. The latter notion is originated from Genette's idea of "timelessness" regarding those "unplaceable" events; it is used by later narrative theorists like Bal. However, the notion of achrony does not address the multiple ordering. We will include more details in a later section. This fourth category thus complements Genette's three categories, forming a coherent set of temporal relations in a narrative. We will use these four categories as our departure point for temporal studies of games.

Previous studies of game time have considered similar ideas yet using different perspectives. Eskelinen's early game studies work contrasts game with narrative. He argues that games only have one necessary time scheme, namely, "the movement from the beginning to the winning or some other situation," and "in cases where another time scheme is invented, it is not as important as the first one" [18]. While the dominant temporal relation in traditional narratives is the one between story time and discourse time, the dominant temporal relation in games, according to Eskelinen, lies between user time and event time. User time is the time taken by the player to perform actions whereas event time is the period for the "happenings" of the game. However, since user time and event time are durations (or time frames) based on one single time scheme, Genette's temporal categories are simply not effective here; hence Eskelinen's discussion of temporal relations only borrows the terms from narratology without the real spirit. Different from Eskelinen, Juul tries to distinguish his study from the narratological approach, even though he agrees that the two approaches are comparable to certain extent. Juul proposes two time schemes for games: play time and fictional time [10]. It is worth noting that in his previous version of this study [19], Juul called fictional time event time, which is coincident with Eskelinen's term. Play time is the time taken by a player to play a game and fictional time denotes the time of the events in the game world. Juul then used the term projection to describe the link between play time and fictional time; however, projection is the one and only temporal relation Juul looks at in games. Later studies follow the same thread but confuse time scheme with time frames (i.e., different periods within one scheme). For example, based on Juul's study, Hitchens extends, modifies, and presents a new model for game time, including playing time, game world time, engine time and game progress time [20]. Tychsen et al. further extend that model, in the context of multiplayer role playing games, into a seven-layer model, including such new layers as server time, story time and world time (derived from the original "game world time"), and perceived time [21]. Another work on game temporality is part of Zagal and Mateas's work for the Game Ontology Project. They proposed four temporal frames for games: real-world time (events taking place in the physical world), gameworld time (events within the represented gameworld, including events associated with gameplay actions), coordination time (events that coordinate the actions of players and agents), and fictive time (applying sociocultural labels to events, as well as narrated event sequences) [11].

A closer look at these works in game studies will tell us that these somewhat arbitrary concepts are not clearly defined. Some works create temporal frames based on one time scheme (e.g., Eskelinen's); some other works create double or more time schemes (e.g., Juul's); yet some create a mix (e.g., Hitchens's and Zagal's). Nevertheless, it is critical 
to be certain about which time scheme the discussion is based upon when setting up a clearly defined model for time. We therefore believe that it is beneficial to return to narrative theory and to start our analysis from the terms clearly defined there and adapt them to games with necessary modifications. In digital games, players do more than reading; instead, they participate in the events in the story and play a part in the telling of the story, too. While story time remains similar, discourse time becomes more complex for games. From the player's perspective, it should refer to both "reading time" and "acting time". Thus, we use the term operation to refer to the running process of a game driven by both the player's actions and the game's autonomous mechanisms. A game story, on the other hand, is the coherent story reconstructed during and/or after the gameplay by the player, which consists of a succession of events in chronological order. Therefore, narrative time in games concerns the relationships between two time schemes: story time and operational time. In our analysis of time in games, we will study the three temporal categories_-order, duration and frequency-based on the relationships between story time and operational time. We will follow some recent narrative theorists (e.g., Bal [22] and Prince [23]) and use narrative speed to replace duration, which makes it more of a relative notion operating between story time and operational time. In addition, we will also study the existence of fuzzy temporality in games to exhaust all the possible temporalities. Although game storytelling is often accused of being simple and linear, we will show the exceptions to this as well as those unique temporal devices games employ.

\subsection{Space in Time: An Integrated Approach to Game Narrative} Space. The approaches to space in narrative theory are not as consistent as those to narrative time because of the lack of a rigorous model like Genette's. Following the approach to time, early narratology works have attempted to make inferences from the relationships between story space and discourse space, but this method did not go too far due to the multiple understandings of what is discourse space. It is unclear whether it is the space on the book pages, the space of the screen, or the space where the act of narrating occurs. When extending the analysis from written narratives to film, Chatman points out that discourse space "can be defined as focus of spatial attention"; "[i]t is the framed area to which the implied audience's attention is directed by the discourse" [24]. In this sense, story space and discourse space simply exist on the same plane. To comprehend the narrative space, the audience works on the mental construction of the storyworld. In digital games, players not only look at the space but also understand the space through navigation and interaction. Therefore, we consider game narrative space the space of the game's storyworld, a term that has been defined earlier in this section. Our later discussion will reveal the characteristics that qualify narrative space in games.

As mentioned earlier, spatial analysis in previous game studies has not gone much beyond classifying the topographic structures of game space. Nitsches' book Video Game Spaces is one of the few exceptions, providing a comprehensive study of game space framed under structure, presentation and functionality [16]. Discussion under "structure" looks at how textual qualities of games are reshaped by 3D game space; topics under "presentation" focus on the roles of moving images and sound in game space; "functionality" addresses the player's interactive access to the game space. By attempting to include all possible dimensions, this book touches on so many peripheral topics that it, in a way, loses its focus when it comes to the ultimate question of how to describe the structural aspects of game narrative space. The key characteristic of game space is that it is dynamic and can be interacted with. Unlike written narratives that can have less precise spatial information and films that can effectively use camera frame to guide the audience's view of the entire space, today's digital games, equipped with more advanced technology like 3D graphics and faster engines, provide expansive visual spaces for players to explore. These spaces cannot be represented without explicit spatial information conveyed via visual, auditory and haptic cues. In a literature of spatial analysis using narrative theory that often treats space as the static "setting" of story, Zoran's work stands out by going beyond this narrow understanding and recognizing that the structure of space influences the reconstructed storyworld in more than one way. In order to discover how the spatial structure affects the storyworld, he goes on to study the inherent structure of space and develops a model that distinguishes three levels of spatial structuring. These three levels are the topographical level (space as a static entity), the chronotopic level (space imposed with events and movements), and the textual level (space imposed with verbal signs) [14]. This model aptly embraces key aspects of narrative space and "anticipates many of the issues explored by subsequent researchers" [15].

There are both parallels and differences between Nitsche's study and Zoran's model. First, they are both created on the premise that understanding space and movement is done through reconstructing the storyworld (i.e., narrative comprehension). Second, the two frameworks overlap in some ways; for example, they both investigate the topographic structures of the space and relations between the textual signs and representations of the space. On the other hand, the most prominent difference between the two works, other than them working with different media, is that Zoran emphasizes the connectedness between space and time so much that he introduces a special level of analysis to track and understand their dynamic relationship. In addition, Zoran's model is solidly built on narrative theory, more clearly defined, and more concentrated on the ways space exists in the reconstructed storyworld. We therefore believe that Zoran's model, though originally created for written narratives, can inform the analysis of narrative space in games. We thus adapt Zoran's model to the interactive context of games and develop three views on game narrative space based on his three levels, which represent three types of inherent structure of space in its three existing modes.

The topographical view, which is Zoran's original first level, treats space as a static entity with fixed spatial reference and separated from temporal reference. Such terms as layout, spatial organization, and spatial structure used in game design 
or game analysis are all related to the topography of game space and thus belong to this view. In this view, maps can be drawn with such geographical reference as locations and landmarks, or references based on other ontological principles like treasure chests. Zoran borrows Bakhtin's notion of chronotope to address the role that time plays in space; hence, at the chronotopic level, space is structured by events and movements, which are all time-referenced. Zoran's level of textural structure investigates how textual (verbal in written narratives) patterns are imposed on the organization of space. In games, where the story is generated on the fly, player actions influence the storyworld through (nonprescripted) events and movements, which in turn causes changes in the on-screen and off-screen spaces (including acoustic space). This calls for a modification of the scope of Zoran's two levels and one possible solution is to define an operational view and a presentational view. In the operational view, the story unfolds (i.e., it is cogenerated by players) over time through events, taking places at one location after another; the space of the storyworld is thus revealed through movements. As a result, game operations impose movement and interactive patterns on the structure of space. In the presentational view, the dynamic presentation of the storyworld imposes its patterns of visual, auditory and haptic cues (i.e., the language of game as a medium) on the structure of space. This distinction between the operational and presentational views helps to isolate issues of how players navigate through and interact with the space from those regarding the visual/auditory display and game interface.

Zoran suggests that there are no clear boundaries between his three levels and in the audience's eyes; "they are always perceived together, one through the other" [14]. Our three views, consequently, are on a spectrum with shifting foci, from the textual (or representational, if we migrate from verbal to visual media) patterns, most immediate to the audience, to the reconstructed world as an existence itself, detached from all the activities that can happen within it.

\section{A Closer Look at Game Narrative Time}

In this section, we examine temporal relationships in games following the most frequently used categories order, speed, and frequency. As previously discussed, these relationships dwell in the dynamics between story time and operational time. We also look at the ways the aforementioned polychrony exists in game storytelling and how it affects the gaming experience indirectly through reducing the linearity and increasing the replayability. In the discussion, we also observe that when integrated into the game mechanics, time can play a significant role in gameplay. This operationalized use of time marks a fundamental difference between digital games and other narrative media.

3.1. Order. In narrative theory, order concerns the relation between the order of events in the presented narrative (i.e., in discourse time) and their chronological sequence in the story constructed by the reader or viewer (i.e., in story time). Correspondingly, in games, order is the relation between the ordering in operation and the ordering in story.
When these two orderings are consistent, we get a linear story. Traditional narratives often play with the sequential ordering to "draw attention to things, to emphasize, to bring about aesthetic or psychological effects, to show various interpretations of an event, to indicate the subtle difference between expectation and realization, and much else besides" [22]. Earlier games were often accused of having stories that were too linear, which can constrain player interactions. However, linear stories and nonlinear stories both have their own disadvantages. As Adams points out, linear stories can have more narrative power and greater emotional impact on players, but the cost is a corresponding loss in player agency [25]. Among the traditional narrative devices to manipulate the order, flashback is sometimes seen in games but flashforward is quite rare. In a 2002 study that analyzed 130 digital games, the result returned a $6 \%$ of the investigated games use flashbacks and 2\% flash-forwards [26]. We believe that the use of these two devices is higher in games that are more recent. Another common nonlinear technique is branching plotlines-commonplace in games and hypertexts because they are easily implemented computationally. As the use of branching plotlines gives the entire game operation multiple orderings, we will discuss it in a latter section that concerns fuzzy temporality.

A good example of manipulation of temporal order can be found in Prince of Persia: The Sands of Time. The game uses voice-over narration to feature the Prince telling a story to a person, whom we will only know at the end. The whole narration is in past tense even though at the same time the player is playing through the Prince's supposedly past adventure. Only at the end-when story time meets with operational time-does the player realize that the whole adventure was narrated in a flashback. Another well-known example of flashback with the player's involvement is the prologue of Max Payne. The game begins with a flashback where the protagonist Max Payne came home three years ago and found his wife and child had just been killed despite the fact that he had chased and shot the killers (enacted by the player). The game then continues with Max Payne carrying out his tasks as an undercover cop to hunt down the Valkry drug traffickers.

A comparison between the two examples cited above shows that the difference lies in the span of the flashback. The duration of the retroversion is an important characteristic of flashbacks. The duration of the flashback played at the opening of Max Payne is only a few minutes, which is typical in film storytelling. The style of the flashback is consistent with the genre of "sophisticated film-noir thriller" and thus helps set the mood [27]. In the example of Prince of Persia: The Sands of Time, on the other hand, the flashback spans over the entire game story; in this case, players, after a little while, will usually start feeling that nothing special is happening, similar to what Bal observes in literary works. Yet in the game, the voice-over narration using past tense is interspersed throughout the entire game reminding players that they are still in the flashback, which creates a hypermediated and slightly cynical or even comic effect. Mechner, writer of this game, openly admits that using voice-over narration as a framing device and nesting 
the entire story in a flashback is a design choice with the intent of combining game genres-making a "survival horror" game ostensibly a "swashbuckling acrobatic actionadventure" [28]. While in both examples the flashback is interactive, in many cases flashbacks can be done in such noninteractive forms as cut-scene, prescripted dialogue, or even on-screen captions. Juul believes that "interactive flashbacks" are problematic because "[ $t$ ] he player's actions in the past may suddenly render the present impossible" [10]. Interestingly, this is exactly the case in Prince of Persia: The Sands of Time. When the player character accidentally dies, the voice-over narration will say: "Wait, what did I just say? That did not happen. Let me back up a bit." The player will then be given another chance to try. This method only intensifies the comic effect of the game narration. To keep the "suspension of disbelief," flashbacks players' interactions are often limited; even if they are not limited, the game may simply reset when players make "illegal" moves.

Moreover, games have one distinct use of temporal order that is not found in other narrative forms, that is, to use order as the answer of a puzzle. Eskelinen gives Doom as an example in this, where the player must find the right event sequence in order to continue [18]. Many adventure games (such as in God of War and Lara Croft titles) contain ordering puzzles - where players need to trigger a set of switches in the right order to open a gate,. Other narratively inflected puzzle games use this mechanism as well, in particular a number of online puzzle games (e.g., Samorost (http://www.amanita-design.net/samorost-1/.)) This practice effectively conjoins the narrative concept of order with the dynamics of ludic play.

3.2. Speed. In narrative theory, speed concerns the relation between the duration of the events that happened in the story and duration of the discourse that tells the story. The narrative speed of a game, correspondingly, is the relation between the duration of the operation of an event and the duration of the happening of that event in the story (i.e., in real-world time). As speed is a relative concept and there is no absolute means to measure it, based on previous theorists, Bal summarizes five canonical tempi that can be used as relative measurements: ellipsis, summary, scene, stretch, and pause, going from fast to slow, respectively [22]. The key to distinguishing one tempo from another is to compare two time schemes, and it does not matter which two they are. Therefore, if discourse time is replaced with operational time, the five tempi can be applied to measure the narrative speed of games.

The most common tempo in games, especially in action sequences, is the scene where events take place in operational time in the same speed as they do in story time. In film, since real people act out the actions in a scene, a perfect "scene" speed can be created; in games, however, where all the actions are computationally presented on the screen, there is not necessarily an absolute "scene" speed. A series of fighting actions, depending on which game is being played, can take slightly different durations. For example, in the 3D game Fable II on XBox360, the duration of in-game fighting actions is roughly the same as that in real world whereas in the 2D game The Legend of Zelda: Phantom Hourglass on Nintendo DS, it feels a lot faster than in the real world as players speed up by tapping the stylus as quickly as they can. The duration of an action sequence is also related to the scale of the game space provided. As long as the sequence takes place within a reasonable range of duration considering the scale of the game, we can consider its speed as scene. Thus, scene is the most used speed when the game progresses in a normal state. Occasionally, we see a game like Animal Crossing, where the game is synced with realworld time so that the game story has the same seasons, holidays and so on as in the real world. Kelly suggests that this game design "intentionally draws on the passage of time to create both emotional resonance and economic value in the gameworld"; this design also encourages players to visit the game storyworld regularly in order to fully experience different events happening at different times of the day and even the year [29].

Summary happens when the duration of an event in the operational time is shorter than that in the story time. This tempo is used when the author wants the time point to make a major leap without showing the details of the happenings in between. For example in Fable II, in the "Birth of a Hero" chapter, the player character grows up into a young adult after a short cut-scene showing the rapid change of seasons accompanied by a voice-over narration; in this way, 10 years of story time is collapsed into 30 seconds of operational time in the game.

The opposite of summary is stretch, when an event takes longer to happen in the operational time than it does in story time. An often-cited example is "bullet time," where the usually very small duration of a bullet's flight is greatly elongated and presented in a slow motion. This effect was made famous by the film Matrix and latter adopted by the game Max Payne. In the game, bullet time goes beyond a representational effect and become an example of game mechanics that gives the player an advantage over enemies. When bullet time is triggered by hitting a key on the keyboard, it slows down the operational time so that the player is able to aim (when shooting) and react (when dodging the bullet). Ruffino comments that the innovative use of bullet time as a game mechanic is "a magnified satisfaction of a yearning that lies behind much computer game playing: the dream of control over time." Yet, it is not just added control, but something more for the player's ludic and aesthetic pleasure; it "transforms fights into quasiballets," where "every step of the fight determines and is determined by the steps of the other "dancers"” [30].

When games use ellipsis, there is a skip of story events in operational time. For example, in Fable II, the player character worked in the Tattered Spire as a labourer for 10 years in an effort to find the right timing to recruit Garth, the Hero of Will. Events happening in such a long story time are presented in a short operational time using ellipsis. The game only selects three moments, from Week 1, Week 38, and Week 137, to present. When the selected events in Week 1 are completed by the player, the game shows the caption "Week 38 " and continues events from there so that things in between are entirely omitted. This tempo is very common 
in games. What is more, the commonplace gameplay device "teleporting" is essentially an ellipsis. When players go to the teleporter, they will be transported instantly to another location.

Lastly, when a story event is paused and the operation is taking care of something else, a pause occurs. This tempo is rarely used in games except in the form of a brief orientation cut-scene in some games. For example, in Prince of Persia: The Sands of Time, there will be a pause when the prince enters a new environment: a quick cut-scene with a camera pan will occur to show players the whole picture of the space and hint to them where the target is. Similarly in Assassin's Creed II, whenever players are about to solve a platforming puzzle in the tomb, a very quick cut-scene will be displayed to show the order of the spatial points players need to be at in order to pull a trigger before the timer is off. In addition, in most games players can pause the game, adjusting game settings or taking a break. This type of pause makes the game more user-friendly, but is not central to the experience or the analysis of game narrative.

3.3. Frequency. Frequency deals with the relation between the number of times an event "really" happened in the story and the number of times it is presented in the operation. The most common relationship is a singular presentation of an event occurring only once. When an event in a story occurs only once but in operation, it is presented more than once, repetition happens. When an event that took place multiple times in the story is only presented in the operation once, iteration happens. Iteration is found mostly in verbal narration, where a repetitive series of events can be summarized verbally (e.g., "for three years, he practices his sword skills every day"). Hence, it is not surprising when we see iterations in the verbal part of the game narration, such as in voice-overs, dialogues and diaries. Aside from being presented verbally, iterative narration is very hard to realize otherwise in a visual medium like film or game, although we do not exclude the possibility of using indirect means to implement iterations. Kinder has done a detailed discussion of iterations in films, where she considers iteration not being necessarily a temporal notion. There are some indirect ways to present iterative events visually in films, which she dubbed "pseudo-iterative." One way is to foreground the singular protagonist within a crowd in a habitual event to imply the iterative nature (e.g., in the hiring scene Bicycle Thief) [31].

Repetition is very common in games, but it is mostly employed as a game mechanic rather than a narrative device. The most common repetition is to help players overcome challenges. When players fail to complete a task, they get to repeat the task until done. In this type of repetition, events in operation may vary (e.g., each fight is different) but story events remain the same. The range of the segment that can be repeated and the maximal number of repetitions allowed are dependent on the design of the game. This type of repetition helps the player master gaming skills but is less relevant to the narrative experience.

Another type of repetition is at the player's choice. In many games, players are allowed to move back and forth. They may revisit a certain game section and repeat what they have done before. In this case, some games are able to offer variations for the repeated section; for example, the enemies may be spawned in different locations than the previous time. This type of repetition due to revisiting adds to a player's experience in both gameplay and narrative.

The third type of repetition is the result of a player's ability to reverse time. In Prince of Persia: The Sands of Time, the Dagger of Time is both a gameplay and a narrative device that can be used by players to turn back time to a point of their choice. At the gameplay level, the Dagger of Time allows players to rewind the game and so redo a task that they failed last time. At the narrative level, the Dagger of Time is so powerful that it can even turn over the story outcome, although in cut-scenes. As part of the prescripted story, after the nonplayer character (NPC) Princess Farah has died and the Dagger has been put into the Hourglass, marking the climax of the game, a cut-scene brings the time all the way back to the beginning, as a result of the Dagger returning to the Hourglass where it belongs. The Prince then starts to tell a story to Farah about the past adventure.

Finally, repetition also occurs at a low level, which can be seen in examples such as an NPCs' repetitive motions (e.g., the forever-swiping motions of the street cleaner in Assassin's Creed II) or a player character's "grinding" activities in order to access a new feature or gain a new status.

\subsection{Polychrony and Narrative Variation. The concept of fuzzy} temporality is developed by Herman to expand Genette's temporal study that is solely based on the relation between story time and discourse time. When one or more events cannot be assigned to an exact position in a story's timeline, it is no longer meaningful to look at the relation between the two time schemes. Inspired by the concept of "fuzzy logic" that opposes the bivalent logic having only true and false values, Herman rejects the bivalence of previous temporal studies that simply consider narrative time to be either determinable or indeterminable. Citing Margolin's notes, Herman states that a given set of events can be ordered in four ways. In the full ordering, for any two events "it is possible to decide whether one is earlier, later, or contemporaneous with the other." In the random ordering, "all mathematically possible arrangements are equally probable." In alternative or multiple ordering, the probability of one ordering can be higher than that of another. In partial ordering, some events can be "uniquely sequenced relative to all others, some only relative to some others, and some relative to none" [15]. Herman goes on to use polychronic narration to entail a system, which consists of three valuesEarlier, Later, and Indeterminate, covering the entire range of fuzzy temporality. In a polychronic narrative, events can be inexactly ordered (i.e., as in the last three types of ordering mentioned above), or inexactly coded (i.e., being inexactly positioned on the timeline), or both.

Herman's discussion of fuzzy temporality can shed a light on temporal studies of games. One key strategy to make narrative interactive- that is, to let players have an impact on the story through interactions-is to generate variations for different readings (i.e., variation in plot) [32]. This is also related to what "breaking the linearity" is about in 
game designers' terms. The most common approach to the creation of a "nonlinear" story is to allow varied orderings to occur for each play of the game. To ensure that the game still follows the overarching story, foldback structure is very popular and is used to balance the freedom at a local level and the overarching narrative at a global level; as Adams points out, it is often adopted by modern storydriven adventure games like The Secret of Monkey Island [25]. In a foldback story, the entire game narrative is broken into several parts (or chapters) and accommodates multiple plot variations. Within each part, players can go through a different set of events and/or in a different order during each play. At the intersections between parts, inevitable events occur. These inevitable events usually follow a fixed order and occur at relatively fixed positions on the overall timeline of the game story. This structure can be seen in such recent examples as Assassin's Creed and Fable II, where the overarching mission or quest is the same, but players have the freedom to pick the side tasks or quests to complete in their favoured order. For "linear" games where there is no narrative variation, because players still interact at a local level, each prescripted (and inevitable) story event possesses a different position on the timeline due to individual pacing of the game. Hence, it is perhaps safe to conclude that digital game storytelling is all polychronic due to its more or less fuzzy temporality. We will explain further through analysis of an example in a later section of this paper. It is also worth mentioning that digital games have unique ways to create narrative variations, or fuzzy temporality in general. Apart from employing temporal devices, spatial structure can also be utilized to facilitate temporal design, which we will discuss in the next section.

\section{A Closer Look at Game Narrative Space}

In this section, we examine in detail narrative space in games from the three views that have been introduced earlier. These three views describe three different modes of how narrative space exists and functions in digital games. To iterate, in the first mode, space is considered as static and independent of both plot enactment and screen representation; it is the topographical structure of narrative space, which forms the underlying spatial reference of the storyworld. In the second mode, space is a space-time complex that encompasses plot enactment, that is, the storyworld as revealed through the operation of the game. In other words, space is structured through events and movements occurring in the operation. In the third mode, space is the presentation of storyworld. The presentational structure determines the visual and auditory manifestations of the game world and shapes players' perceptions and thoughts. Although these three modes are differentiated for the purpose of analytical clarity, we need to remember that it is the combination of the three modes working together that make the design and experience of a game's narrative space effective and satisfying.

4.1. Topographical Structure. The topographical view treats space as a static entity with fixed spatial reference and separated from temporal reference. The topographical structure can be perceived as a map or any mental conception that features the spatial relations between locations or entities. A map of a story certainly cannot exhaust all the topographical information; so "blanks" are unavoidable. It is often up to the audience whether the picture of the storyworld is clear; when it is unclear, they will attempt to fill in the blanks with imagination. As Zoran suggests, the mental conception can include not only locations but also the quality of things based on different ontological principles; in this case, "landmarks" and "regions" can be used to mark important locations or areas. Hence, there are all sorts of readerconstructed cognitive maps for reading purposes as well as in-game or player-drawn game maps and sketches aimed at enhancing player performance in different ways. Among all the characteristics relevant to topographical structure, layout and oppositions are two major considerations for structuring the space.

4.1.1. Topographical Layouts. The study of space has always involved typologies of spatial models based on topographical features. This is important because "[e]ven if players gain access to the space-generation process, some structure has to be provided either from the player or the system" [16]. Indeed, for interactive narratives and games, the discussion and classification of spatial models is always related to spatial navigation. For example, Murray discusses two structures of spatial navigation for interactive narrative: the maze and the rhizome [33]. Nitsche also proposes several distinct spatial forms: tracks and rails, labyrinths and mazes, and arenas. Since these forms define the spatial logic in their own way, their structures shape paths, edges, and regions, which in turn determine the ways of player navigation [16].

In the game design field, the layout of the game space is often created as part of the level design. Adams considers a successful layout needs to be "appropriate for the storyline and to achieve the atmosphere and pacing required to keep players engaged in the game world" [25]. Adams gives a list of 7 common patterns of layouts that we believe to be a practical typology of game spatial layouts. An open layout represents the outdoors and gives the player the freedom to wander about. When the player goes indoors or underground, as Adams observes, the layout often switches to a network or combination layout. Examples of open layout are role-playing games like the Fable series, war games like Battlefield 1942, and action adventure games like Assassin's Creed. The settings mimic their corresponding worlds in real-life and thus have few, if any, visible spatial boundaries. A linear layout is not bound to any particular shape, but it does ensure a fixed sequence for the player to experience. This is similar to Nitsche's concept of tracks and rails [16]. A parallel layout is a variation of the linear layout. It is like tracks with switches that allow the player to switch from one track to another. A ring layout makes the player's path return to the starting point, which is often used by racing games. A network layout provides more ways of connecting spaces and gives the player more freedom compared with a layout with tracks. A huband-spoke layout starts the player from a hub in the centre. The player can go out of the hub to a space but will have to return to the hub before heading out to another space. 
Different layouts provide different qualities for player navigation. For instance, the hub-and-spoke structure is claimed by game designer Steve Gaynor to be "the most common high-level organizational strategy". He cites the Medical Pavilion hub in BioShock as an example of such structure, where "minor spaces are always closer to major spaces than they are to other minor spaces-the player always passes through the hub to get to another spoke" [34]. In contrast to a full open layout, the hubs and spokes give the player reorienting spatial references so that the comprehension and thus navigation of the game space become easier. Adams also reminds us that designers are not confined to just one layout for their game space. Some games have a combination of various layouts, either at the same level or in more complexly nested combinations across multiple levels.

4.1.2. Spatial Oppositions. In a thoughtful narrative design, spatial oppositions of all sorts can be used to structure the story world and thus create the desired story effect. In her discussion of location as a fabula element, Bal stresses that "[o]ppositions are constructions; it is important not to forget that and "naturalize" them" [22]. Similarly, Zoran considers that the map of a topographical structure "is based on a series of oppositions" [14]. Spatial oppositions are typically physical, for example, inside and outside, center and periphery, city and country, and so forth $[14,22]$. They can be endowed with meanings or experiences, following, creating, or playing with conventions. In the Assassin's Creed series, for example, the rooftop space is open and relatively safe since the protagonist is out of sight of most of the guards whereas in the ground-level space he needs to be careful about his actions; players thus can take advantage of this opposition-based spatial convention to adjust their own pacing. This example illustrates how the design of narrativized space can affect ludic play.

Other than shaping the gameplay experience, spatial oppositions can also offer a way to group narrative elements and thus simplify complex content for players. Let us take a look at the map of Masyaf town (Figure 1) in Assassin's Creed. The upper part represents the mountain fortress and the lower part the village. Aside from being geographically separated, these two spaces also contrast with respect to population and infrastructure density. Naturally, in the village players will raise their alert level when surrounded by villagers as well as soldiers. In contrast, the mountain fortress serves as a home region where the player just gets instructions and training. Thus, the convention created via spatial opposition helps players adapt to the environment more easily and adjust their alert level and pacing accordingly. Again, the narrativized space serves to nuance ludic decisionmaking. It is worth noting that this type of opposition is reused in Assassin's Creed II, between the Monteriggioni villa, serving as the home region, and other cities, although on a much larger geographical scale.

The boundary or the transitory place between two opposed locations often functions as a mediator, as Bal also suggests [22]. In Masyaf, the passage between the mountain fortress and the village is the gateway for the player avatar to

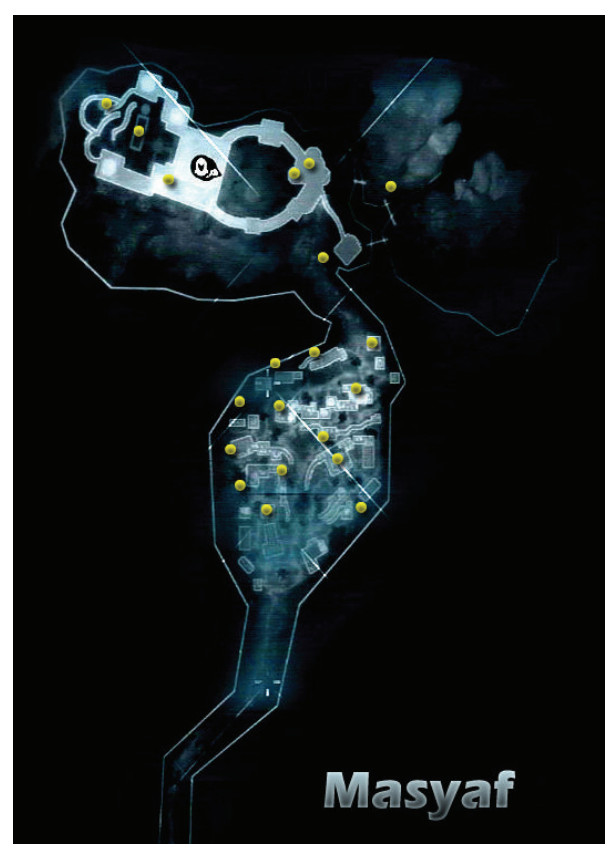

Figure 1: Masyaf town in Assassin's Creed (source: Ubisoft, 2007; image from IGN.com, used with permission).

step into the other part of the game world, either reporting to his master or going to carry out his missions. On this passage, the player often gets instructions from an NPC. A transitory place allows the player to take a break between missions (or levels) and get ready for the next adventure that will take place in yet another location in the game world.

4.2. Operational Structure. As defined earlier, the operational view sees the storyworld revealed through events and movements over time while the story unfolds. The operational structure is formed by characteristics that shape game spatial operations by regulating and patterning movements. The view of operational structure corresponds with Zoran's level of chronotopic structure, which addresses "what may be defined by an integration of spatial and temporal categories as movement and change" [14]. The chronotopic structure has a significant impact on the plot, which is not just a temporal structure but also includes "routes, movement, directions, volume, simultaneity, and so forth" [14]. In games, since the protagonist is often (en)acted by the player, the shaping of the plot is dependent on both predesigned plot structure and the ways the player navigates and interacts. In this sense, game plot becomes a set of all possible narrative instances, consistent with Manovich's conception of a "database narrative" [35]. We call this set of plot instances a plot set. When looking at the game retrospectively after one play, the player has gone through only one instantiation of the plot set. We thus consider this traversing act as an operation to differentiate it from chronotope. This section will explore the spatial qualities that characterize the operational structure of narrative space in games. One of the fundamental characteristics is the mobility of characters and objects, which is the key factor to identify a change 
of the state of the plot. Other characteristics characterize movements over the course of the operation. Our approach to the operational characteristics is inspired by Zoran. He suggests that synchronic and diachronic relationships are the two main concerns for the level of chronotopic structure. The former detects motion and rest whereas the latter characterizes the movement through directions, axes, and powers. Here we selectively adapt his suggestions to games and draw in some considerations specific to games as well.

4.2.1. Mobility of Characters and Objects. At any given moment, characters and objects in a game are in one of the two spatial states: movement or rest. Here the focus is on their general mobility. Some characters or objects move between spaces while others stay in one space. Hence we can replace the account of mobility with a question like "what is attached to one particular space, what not?"

Those characters attached to one space become the "background" of the space, that is, part of the context, especially when not interacting with the player. In this case, characters play the same role as other environmental objects. When players interact with these characters, the plot can change locally. The range of a character's mobility and interactivity often determines the significance of the change. Many NPCs are designed to attach to one location or one subspace. They are either enemies or background characters. This is very common in a game like Fable II, where, for example, the archaeologist can always be found in the same spot and the blacksmith always belongs to his shop. For "attached-to-space" characters, their qualities can be coordinated with the space. For example, on a difficult game level, an enemy character can be tough and hard to defeat.

Characters that are able to move with greater range from one space to another can play a more significant role in plot development. Hence, they are often the main characters that grow with the plot, along with the player character. The dog in Fable II is such a character that follows the player avatar all along. In general, the more highly mobile the characters, the more complex the plot is. In Heavy Rain, all four main characters are playable and highly mobile, which makes the plot intriguing and full of suspense. Similarly, some objects in games that have a high mobility can play an important role in the game operation and thus the plot development. For example, in Prince of Persia: The Sands of Time, the Dagger of Time is such an object with mobility. Once obtained, it follows with the player avatar and performs the magic of time reversal on request. The use of the dagger by the player contributes to the construction of the plot.

There are also characters and objects that are mobile within a certain range. Horses in Assassin's Creed series are one such example. They are located outside city gates and can be ridden by the player avatar all around in countryside but not into the city. In this way, they speed up the protagonist's movements between cities and yet make the scale of distance feel real.
4.2.2. Paths and Axes. A path between locations can be unidirectional or bidirectional; in the latter case, the movement is reversible. Axes are the principal paths surrounding which major events and actions take place. Axes help organize narrative content and form the operational structure for the space, especially for those plots that are driven by character movements.

For role-playing games, while players must move along the axis in order to progress in the game by pursuing the main quest, they are also allowed to explore the world and make social interactions on side quests that follow paths branching out from the axis. This is often realized in a rhizome structure, which is a "tuber root system in which any point may be connected to any other point" [33]. The tuber represents the axis, or the path for the main quest whereas the roots are the paths of other side quests. For example, the role-playing game Planescape Torment, as Diane Carr finds, uses such structure that "sends its multitasking players in rhizomic circles, deviations and side-quests in search of lost memories and fragmented histories" [36]. Such operational structure featuring one axis is, in a strict sense, still a linear structure. As Carlquist observes, even given all the player choices, games like Planescape Torment are still linear games because the player "still has to follow the main plot in a certain way" while pursuing the main quest in a linear order [37]. Fable II is another role-playing game that follows the rhizome structure.

The mobility and interactivity of characters and objects, directions for paths, existence of axes, as well as other spatial features work together to form the operational structure for games. They construct the storyworld for the player to navigate through and interact. The game mechanics are also embedded within the same structure, which therefore supports both narrative experience and ludic experience. This sharing of the operational structure between narrative and game mechanism allows for the deeper integration of game story within the gameplay.

4.3. Presentational Structure. The notion of presentational structure is mainly derived from Zoran's level of textual structure. Here we extend the scope of narrative text to cover visual narratives including films and games. The presentational structure concerns how the patterns of visual, auditory, textual, haptic and other cues (i.e., the language of game as a medium) are imposed on the dynamic presentation of the storyworld. Hence, the analysis on this level focuses on the ways and materials through which the game world is presented, as well as how the presentation incorporates player actions. The presentational view of game narrative space contains a large variety of aspects ranging from the organization of information to low-level representational techniques. These aspects have considerable commonality with those of films.

4.3.1. On-Screen and Off-Screen Space. While the topographic and operational structure can reveal the major spatial reference and movement potentials of the game world, detailed information on the space remains unclear to the player in these two views, and needs to be structured 
during the presentation of the game. The selection of the spatial information to reveal on the screen is probably the first thing at issue when presenting the story space. For complex games with a 3D story world, the on-screen space is different from the "shot space" of a film although it applies many cinematic techniques. In films, the view is constrained by the laws of optics and physics; in games, the view is computational and dynamic. The game's screen image is depicted by a "virtual camera," which extends far beyond the functionality of an actual camera. For example, a virtual camera can follow fast moving objects to create the "bullet time" effect, which manipulates not only the perceived space but also the perceived time of the player. Fly-through, originally used in the field of architecture, is yet another virtual cinematic technique to give the player a view of the space ahead of time from a predefined perspective, where the camera is "flying" or circling around the space. Prince of Persia: The Sands of Time and Assassin's Creed II both use this technique extensively. Another different use of on-screen space of a game is to display the screen interface of the game, which will be discussed latter.

For games that limit or disallow players to change the camera view, the on-screen space can be seen as a relatively independent space. In this case, it is close to the "shot space" of a film, where the screen composition conventions from film are selectively applied. In the controlled presentational space, the on-screen and off-screen spaces are not necessarily connected. In God of War, the camera angle will usually adjust or follow when the player character enters a less visible spot. When the player avatar enters a new subspace, there is usually an establishing shot of the space and then the camera closes up. Shot/reverse shot, a common camera convention used for two-person scenes, however, is only selectively employed by God of War: it is used in conversations but not in most of the fight scenes. In many combat scenes the on-screen space is enclosed with clear boundaries and thus cut off from off-screen space; thus, the on-screen and off-screen spaces are not necessarily continuous. Another example is Heavy Rain, which features discontinuous or limited continuous space because the on-screen space is heavily "edited" with restricted player control of the camera angle. In these cases, the designers need to decide how to transition from one on-screen space to another, which we will discuss in the following subsection.

4.3.2. Acoustic Space. While visual factors dominate the presentation in many games, in others auditory factors play an equally important role in the construction of the game space. As in film, sound is used to set the mood, create tension, and help to enhance the realism of the game world. In Fable II, the ambient sound helps to define the environment and shape the emotional tenor of the progress through the game space. When the player avatar is exploring a town, the background music is quiet and peaceful. When he or she is on the road or in the cave where bandits and monsters are nearby, the music becomes loud and ominous. When combat begins, drumbeats kick in to intensify the fighting mood. This dynamic design of musical soundtrack effectively creates both narrative and gameplay tensions that intensify the player's experience of the game.

Game sound also has functions unique to the medium. For example, sound provides feedback for player interactions (e.g., footsteps made by the player avatar) and hints for players' next moves (e.g., a sound coming from off-screen suggesting the direction of an approaching enemy). Such auditory cues help players perceive the 3D game space and imagine the off-screen space. Grimshaw and Schott maintain that "sound functions as an acoustic ecology" consisting of both the players and their soundscapes. In their study of such ecology of first-person shooters, they discover that sophisticated use of sound "aids in imaginative immersion through the provision of virtual resonating spaces and paraspaces" [38]. In music/rhythm games like Rock Band and Dance Dance Revolution, sound goes even further to become part of the core gameplay mechanic.

4.3.3. Spatial Segmentation. In the operational view, game space is structured and presented in a temporal continuum. How spatial information is segmented and how the player gets passed from one segment of space to the next are two questions relevant to the spatial organization. What is more, according to Zagal et al., spatial segmentation "results from the division of the gameworld into different spaces that also partition gameplay" [39]. Although Zagal's original study is based on vintage arcade games, we find the notion applicable to digital games in general. Since digital games have overcome technical barriers and evolved from earlier 2D platforms to current complicated 3D game worlds, setting the boundaries becomes an important part of the design strategy to create rich gaming experiences. Hence, a game world is divided into distinct subspaces, each of which has different spatial features or even game rules. The question of how to segment the space also triggers the question of how to make the player have a fluid experience navigating through the subspaces. When the subspaces are disconnected topographically, they can be displayed in the form of episodes or scenes cut from one to the next; otherwise, they may be connected and form the entire game space in the structure of a tree or a network in graph theory terms, or, a maze or a rhizome in Murray's terms [33]. In the latter case, players simply "walk" into the next subspace.

There are four common styles for the transition from one subspace to another that is not directly connected: direct cut, fly-through (or orientation cut-scene), cut-scene, and caption. In Prince of Persia: The Sands of Time, for instance, when finishing one session, the player-avatar is often simply brought to a new setting to start a new session; this transition is a direct cut. Sometimes to make up for the possible loss of orientation, the game will play a "flythrough" video sequence to familiarize the player with the new subspace. Cut-scenes are another transitional means prevalent in games where a video sequence is played to introduce background information. Captions are often seen in text heavy games that simply tell players they are to enter a new subspace. Fable II is one such example. Another function of cut-scenes and caption screens is to occupy the player 
during the game loading time, which is often needed for transitions between spatial segments.

4.3.4. Perspective. "Perspective" exists in any form of narrative. The notion of perspective, or point of view, can refer to two possible layers of meaning. One is the psychological point of view that locates attitudes and emotions; the other is the optical point of view that refers to the visual positioning of the frame. The source of the perspective in both cases can be either subjective (from a particular character) or objective (from an external narrator or a neutral viewer). The psychological and visual layers are often intertwined-a "first-person" visual can reinforce the psychological perspective of the protagonist. The points of view of different sources can structure the story world on the presentational level. In films, camera angle and optical point of view can be used to reinforce either subjective (character-driven) or objective (neutral and observational) psychological perspectives. For games that employ virtual cameras, the player is simply assigned either the protagonist point of view as in first-person games, or a bird's eye view as in third-person games. It is very hard to find an instance in games where the psychological point of view is separated from the physical one, a case that is common to films. Even when the two perspectives are separated, it is often realized through voice-over narration. In Prince of Persia: The Sands of Time and Max Payne, for instance, the player controlled protagonist would occasionally talk to the player and comment on what has happened.

Unlike film viewers, game players sometimes are given the freedom of shifting their camera view. In Prince of Persia: The Sands of Time, the third-person camera view is fully controlled by the player and it can be switched to the player avatar's first-person view. Having the two kinds of view Prince of Persia possesses, Assassin's Creed also has an "eagle vision" as an option that offers the player a filtered vision that foregrounds the identity of targeted characters (e.g., guards or informants). As Adams summarizes, the firstperson perspective is only used by avatar-based games, where the camera assumes the position of the avatar's eyes. The third-person perspective, most prevalent in 3D action and action-adventure games, has a more flexible camera view. In a normal state, the camera follows the avatar at a distance from a slightly higher angle; during combat, the camera cranes up and tilts down to enable the player to see more of the environment, as in the case of God of War. The aerial perspectives feature top-down camera views from different angles and are widely adopted by party-based battle games and strategy games [25].

Other than presenting players the view based on their avatars' in-game movement, the camera can also contribute to the game's interactive mechanism by guiding the player's attention to those interactive elements. This is usually done by a direct cut to a zoom-in view of an object or a space, implying what or where the player's next step is to be (e.g., picking up an item or going to that space).

4.3.5. The Screen Interface. For film, the screen is a presentational medium. For games, the screen has a second role- the interface between the game and the player. In our terms, the game's screen functions on both the operational level (supporting navigation and decision making) and the presentational level (presenting the game and its space as it is dynamically traversed). This duality corresponds to Manovich's distinction of the interactive screen as both a control device and a presentation device [35]. This double role can complicate the screen, layering the spatial information with interface information (control menu, dialogues, character status, game statistics, help information, etc.). The layering of information is realized in a screen layout that takes the form of a windowed view, an overlaid view, or a combination of the two, as Adams observes [25]. In a windowed view, the main window displays the game world, while interface information is shown in a separate window on the side, the bottom or the top of the main window. Many strategy games and some role-play games use this layout. This layout can potentially lessen the sense of immersion but it delivers interface information in an easy way. In an overlaid view, the interface information is imposed onto the main view in an opaque or semitransparent fashion. Aside from the two ways Adams observes, there is the third way to enable the screen to assume the double role. For games that strive to provide an immersive environment, they present a relatively clean full screen view displaying the game world but are willing to switch to a menu view at the player's request. Assassin's Creed and Fable II, for example, both have such interface for players to check maps and resources.

Besides the above use of the screen, there is another technique-split screen - that presents multiple spaces in the same screen. Split screen is not uncommon in modern films. It is typically employed when two or more characters take actions simultaneously; they can be in different places or the same place but viewed from different perspectives. Heavy Rain, for instance, uses split screen for such occasions. Split screen is also used in multiplayer games. Wolf's spatial structure type 10-"multiple, nonadjacent spaces displayed on-screen simultaneously"-refers to this technique [4]. He cites the racing game High Velocity as an example where players can see both their own view and the opponent's view on-screen. This helps players strategize their moves although it might distract their attention in the meanwhile.

\section{Time, Space, and Plot: From Analysis to Design}

In previous sections, we have developed a rich descriptive framework for analyzing time and space in game storytelling. How players experience a game is always influenced by the way the game is designed, and how players interpret a game story is always influenced by the way the game plot is organized and arranged. Time and space converge in the plot and together shape the structure of the story experience. Bridgeman describes how we perceive a plot: "our image of a work can involve the paths of the protagonists around their world, bringing together time and space to shape a plot" [2]. As we have discussed in a previous section, the plot of a game is an instance of the plot set that players go through during one play. On first examination, game plot might be 
seen as a function of time since, like a flowchart, it consists of events connected with decision points. In his discussion of interactive narrative, Meadows relates the notion of "plot" to the concept "use-case scenario" in software engineering, which consists of "the function, flow, time, and interaction between a user (or reader) and a particular piece of software" [40]. There are four interactive narrative structures most often cited by game designers, (admittedly without hard and distinct boundaries between the categories): the linear structure, the branching structure, the foldback structure, and the open structure [25]. The linear, foldback and open structures also correspond with Meadows' nodal, modulated, and open plot structures of interactive narratives including games [40].) These four narrative structures in turn support four structures for the gameplay and associated temporal design. All games' interactive narrative structures encompass polychrony-the key to making the story interactive-in one way or another. The fuzzy temporality brought by polychrony helps create interactive space: the indeterminate parts on the timeline are the gaps for players to fill and to create their emergent narrative. For example, in Grand Auto Theft III, as Rouse observes, although the main narrative arc consists of prescripted missions and cut-scenes, players are allowed to choose the missions they want to complete and follow their own order to complete them [41].

The above classification of structures frames the plot set in a collective view of an interactive narrative. From the audience or player's point of view, each reading or play is an integral experience of one plot instance. While most plots are constructed by a sequence of events that follow a temporal order or causal logic, other sequencing orders are possible. For examples, the events can be sequenced based on thematic changes, or on the portrayal of one key character after another, or based on one location after another. Nonetheless, no matter what principle is at work for the sequencing of events, many narratives carefully sequence and time the story events so that the dramatic tension is built into a strong emotional arc. These plots are based on the classical Aristotelian model and consist of three acts, which typically follow a rising action, a climax and a falling action, forming the dramatic arc. The dramatic arc seems to "achieve its effects without the audience's conscious awareness" [42].

In interactive storytelling systems, designers and developers use planning algorithms to sequence events based on their tension levels in order to ensure a strong dramatic and emotional tension arc. In the interactive drama Façade, the plot is divided into two levels of units. On a high level, the drama manager sequences a bag of dramatic beats based on the causal relation between major events. Façade's beats are crafted based on traditional dramatic writing-where the dramatic beats represent the smallest units of dramatic action. On a low level, each beat contains a bag of joint dialog behaviors (jdbs); in response to player interactions, the beat dynamically selects and sequences a subset of jdbs. The system keeps track of the tension value for each beat and selects the next unused beat with the right tension value as well as other preconditions to ensure "an authorspecified story tension arc" [43]. While some interactive narratives like Façade maintain a high level of authorial control over the plot, other systems focus more on player characters' goals and actions. Barros and Musse created a prototype interactive storytelling system called Fabulator, which is based on the "riddle" master plot [44]. Their tension arcs model assumes that the tension will rise when the player acquires more knowledge to move closer to the truth. The plot climaxes when the player is just one clue away from solving the riddle and ends when the riddle is solved. In order to shape the tension arc to the desired level, the system dynamically adjusts the level of difficulty through participation by NPCs (i.e., helping or not helping the player character) [44]. To cater more to the player's preference, Thue et al. propose a player modelling approach via their interactive storytelling system PaSSAGE [45]. Their system creates the player model by automatically learning the style of play preferred by the player. It then uses the model dynamically to select the events and deliver an adapted story. To ensure the dramatic arc, events are grouped into phases of Joseph Campbell's Monomyth structure so that at runtime the system will select an event from the right phase.

As discovered in our spatial analysis, the spatial layout of the storyworld also affects how players traverse the game because the spatial structure is imposed on players' experience of time (e.g., one needs to reach room A via room B). This is especially true for spatial stories (or travel narratives) that are driven by a character's movement across a map. For spatial stories, as Jenkins argues, “organizing the plot becomes a matter of designing the geography of imaginary worlds so that obstacles thwart and affordances facilitate the protagonist's forward movement towards resolution." In this way, spatial exploration aids in the plot exploration. For games featuring spatial stories, successful plot design goes beyond the formulation of a sequence of events. Rather, it is based on the creation of a "compelling framework" where players can have some freedom at a local level without "totally derailing the larger narrative trajectory." As Jenkins rightfully points out, in order to more effectively reach this goal, game designers need to "develop their craft through a process of experimentation and refinement of basic narrative devices, becoming better at shaping narrative experiences without unduly constraining the space for improvisation within their games" [46].

While a game plot can be organized with certain temporal and spatial patterns at a high level, it can be shaped at a low level as well. For example, at the high level of a game, the sequence of inevitable events shapes the dramatic arc warranting an overall narrative experience; when these events are bound to specific locations, the spatial structure becomes part of the plot engineering, too. For example, in PaSSAGE, the plot sequencing takes into consideration the character's location in the game space. When the next event is chosen based on the player model, the system manages this selection through triggers. The triggers monitor the game environment and search for the suitable actors within a reasonable distance of the player's current position [45]. At a low level, a plot decision point located between two events can be designed as either a temporal transition or a spatial one. A temporal transition can use one of the four styles discussed in the subsection of Spatial Segmentation whereas 
a spatial transition can use forking paths, bridges, elevators, and so forth in the game space, just like any transition in the real physical space. In an interactive narrative that is presented in a realistic game world, each event can be bound to specific time and space constraints, instead of appearing in a random fashion. There are two ways to handle these constraints. One way is to build these constraints into the sequencing mechanism (such as in the aforementioned PaSSAGE example) so that the system makes sure of the "eligibility" of the upcoming event. The other way is to design a flexible "transportation" mechanism in the game space so that the player can easily "jump" to the next location following a trigger event (such as in Fable II). Most games employ a combination of the two ways.

The design choices for temporal devices and spatial characteristics work together to support, inflect the storytelling style and shape players' narrative experience. The descriptive framework developed earlier in this paper has enabled us to see many techniques and devices within existing narrative designs as well as related gameplay design decisions. To make the connection between analytical finding and narrative design more visible, the following two tables summarize the key concepts we have discussed and their application and function in games. An important note about the tables is that they are not intended to include all concepts we have touched on in this work, let alone all applications in the vast field of digital games.

Tables 1 and 2 provide a high-level overview of the concepts we have explored in this work. We recognize that there are many nonnarrative devices and characteristics that are not included in our discussion. Because the focus of our work is the structure of digital game storytelling, we have limited our discussion to the narrative perspective. Although the use of time as a narrative device is not as rich as in traditional narratives, time is used in multiple ways as a significant and expressive gameplay device. Granting control of time to players has various effects in the gameplay and the game experience. It helps players overcome challenges and creates fun by allowing players to rewind the game (e.g., Prince of Persia: The Sands of Time, Max Payne), magnifying the fighting process (e.g., "bullet time"), speeding up the transportation (e.g., "teleporting"), giving hints on puzzles (e.g., orientation cut-scenes for platforming puzzles), and so forth. There is also a time-based device very common in games-the ticking clock, a term proposed for games by Wolf [47]. This device intensifies challenge by setting a time limit for players to finish a task or an action sequence. The tensions that the "ticking clock" creates also shape players' emotional experiences. Similarly, space can be used in many creative ways in gameplay. For instance, space plays a very active role in game puzzles. To conclude, by focusing on the narrative comprehension and experience, our approach generates rich insights for game narrative design.

\section{Review through a Case Analysis}

In order to fully understand how temporal relations and spatial characteristics act together to structure a game plot and shape narrative experience, it is best to examine a game example in detail and conduct an in-depth analysis. In this section, we use one game level in the historical action adventure game Assassin's Creed to illustrate how time and space are structured in game storytelling. We pick an action adventure game for several reasons. Adventure games are famous for their rich stories. Mallon and Webb's empirical study on adventure games shows that a good adventure game demands more of well-designed narrative structure [48]. Adams points out that the designers of adventure games need to "bring not just a story but a world to life-a world in which a story is taking place" [25]; hence the spatial design is crucial to the story. Compared with pure adventure games, action adventures are faster paced and thus players feel more time pressure; therefore, more considerations of temporal design are required as well. We believe that Assassin's Creed's open world structure is a good example for the examination of how time and space are designed in games via a variety of devices and techniques.

Since games are essentially complicated systems presenting content hierarchically, both temporal relations and spatial characteristics exist in games at multiple levels. Therefore, we need to decide on the level of granularity for our case analysis. Zagal et al. suggest that a game ontology should start in a "middle-out" fashion because the "most readily observable" categories tend to exist in the middle of the ontology; in this way, both more abstract and more specific concepts can be brought out latter by refined analysis [49]. Similarly, in order for our case analysis to inspire both more abstract and more specific insights, we start out from one specific game level that is easily distinguished and observed by both temporal and spatial standards. According to Zagal et al., a level in a game is "a recognizable subspace of the gameworld". Although level can refer to the degree of difficulty as well, "[w]hat helps distinguish a level from other forms of spatial segmentation is the discontinuity in gameplay and in space between one level and another; the more evident the discontinuity, the greater the notion of level" [39]. In our paper, we use this definition for game level. We thus choose the Damascus Poor District in Assassin's Creed's Memory Block 2 as an example game level to demonstrate how to look at the time and space in a game narrative. The entire game consists of seven "memory blocks" that can be seen as seven chapters. These blocks are not to be confused with game levels since they are not based on game space. Instead, they are based on temporal sequence, so each memory block can contain events occurring in different spaces. Each block has a set of objectives for players to finish through four types of activities: assassination, scaling viewpoints, investigations and saving citizens. Each activity is enveloped in a "memory strand"; once played, the content of the strand can be viewed in the main menu. In the pause menu, players can check on the game status as well as access the map. Figure 2 shows the in-game map of Damascus Poor District, which is the level of our analysis.

6.1. Temporal Analysis. The story of Assassin's Creed overarches the game through the seven memory blocks. On the highest level, the story is presented in a foldback structure, 
TABLE 1: Summary of temporal devices discussed that affect narrative and gameplay experience.

\begin{tabular}{|c|c|c|c|c|}
\hline $\begin{array}{l}\text { Devices for game } \\
\text { narrative time }\end{array}$ & Main forms in games & $\begin{array}{l}\text { Function in game } \\
\text { narrative }\end{array}$ & Function in gameplay & $\begin{array}{l}\text { Examples cited } \\
\text { (selective) }\end{array}$ \\
\hline $\begin{array}{l}\text { Order } \\
\text { flashback }\end{array}$ & $\begin{array}{l}\text { Dialogue, cut-scene, } \\
\text { screen caption, text (e.g., } \\
\text { diaries, logs) }\end{array}$ & $\begin{array}{l}\text { Provide information; } \\
\text { stylistic/genre } \\
\text { storytelling }\end{array}$ & $\begin{array}{l}\text { Game mechanic (e.g., } \\
\text { Dagger of Time) }\end{array}$ & $\begin{array}{l}\text { Prince of Persia: Sands } \\
\text { of Time; Max Payne }\end{array}$ \\
\hline $\begin{array}{l}\text { Speed } \\
\text { scene }\end{array}$ & $\begin{array}{l}\text { Appeared in the normal } \\
\text { state }\end{array}$ & Create realism & Create realism & $\begin{array}{l}\text { Fable II; Animal } \\
\text { Crossing; Assassin's } \\
\text { Creed }\end{array}$ \\
\hline $\begin{array}{l}\text { Speed } \\
\text { summary } \\
\text { ellipsis }\end{array}$ & $\begin{array}{l}\text { Cut-scene, voice-over, } \\
\text { screen caption; Direct cut, } \\
\text { screen caption }\end{array}$ & $\begin{array}{l}\text { Increase storytelling } \\
\text { efficiency }\end{array}$ & $\begin{array}{l}\text { Increase gameplay } \\
\text { efficiency }\end{array}$ & $\begin{array}{l}\text { Fable II; Assassin's } \\
\text { Creed; Max Payne }\end{array}$ \\
\hline $\begin{array}{l}\text { Speed } \\
\text { stretch }\end{array}$ & Cut-scene, slow motion & $\begin{array}{l}\text { Stylistic/genre } \\
\text { storytelling }\end{array}$ & $\begin{array}{l}\text { Game mechanic (e.g., } \\
\text { Bullet Time) }\end{array}$ & Max Payne \\
\hline $\begin{array}{l}\text { Speed } \\
\text { pause }\end{array}$ & $\begin{array}{l}\text { Orientation cut-scene, } \\
\text { pause menu }\end{array}$ & N/A & $\begin{array}{l}\text { Orientation; menu } \\
\text { option }\end{array}$ & $\begin{array}{l}\text { Prince of Persia: Sands } \\
\text { of Time; Assassin's } \\
\text { Creed }\end{array}$ \\
\hline $\begin{array}{l}\text { Frequency } \\
\text { repetition }\end{array}$ & $\begin{array}{l}\text { Task repetition when died } \\
\text { or failed; game replay }\end{array}$ & $\begin{array}{l}\text { Create narrative } \\
\text { variation }\end{array}$ & $\begin{array}{l}\text { Ease the challenge; help } \\
\text { mastering the skill }\end{array}$ & $\begin{array}{l}\text { Assassin's Creed; } \\
\text { Prince of Persia: Sands } \\
\text { of Time }\end{array}$ \\
\hline Polychrony & $\begin{array}{l}\text { Appeared in all plot } \\
\text { structures of games }\end{array}$ & $\begin{array}{l}\text { Create narrative } \\
\text { variation; increase } \\
\text { interactivity }\end{array}$ & $\begin{array}{l}\text { Create gameplay } \\
\text { variation; increase } \\
\text { interactivity }\end{array}$ & $\begin{array}{l}\text { Assassin's Creed; } \\
\text { Grand Theft Auto III; } \\
\text { Fable II, and so forth }\end{array}$ \\
\hline
\end{tabular}

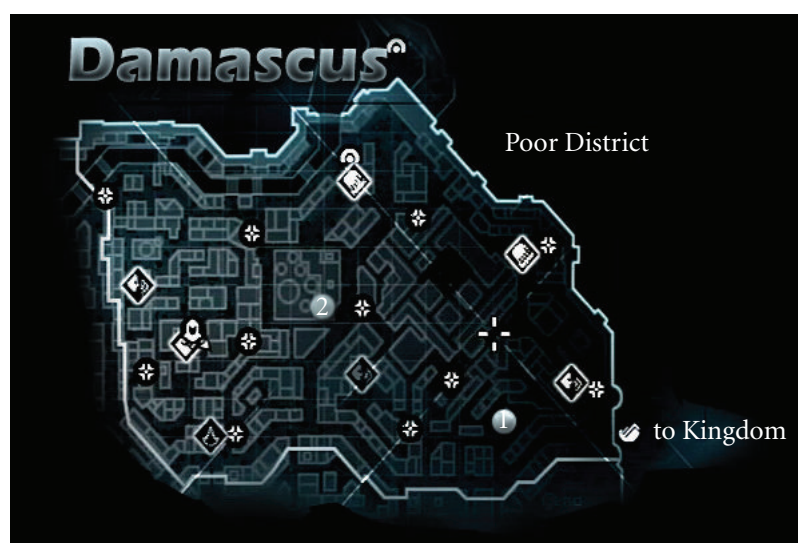

Figure 2: Damascus Poor District in Assassin's Creed (source: Ubisoft, 2007; image from IGN.com, used with permission).

where the ordering of the main sequence (from memory block 1 to 7 ) is fixed but the ordering of events within one block is not totally predeterminate. Overall, most parts of the story are presented in the scene tempo, which resembles the real-world speed. On the highest level, the repetition is realized through replay. Once a memory block is complete, it becomes replayable; however, one play of a block can be different from another in terms of the selection of activities to do and the ordering of doing them. In the following, we present a temporal analysis of Damascus Poor District in detail; hence, we will discuss the phenomena within the game level. In the Damascus Poor District level, investigation includes three subtypes: pickpocketing, eavesdropping, and interrogation.
6.1.1. Order. Consistent with what we have discussed earlier, flashbacks (retroversion) appear here and there mainly in a typical form of dialogue sequence (cut-scene) but flashforwards (anticipation) seem to be nonexistent. The most prominent example is the eavesdropping activities. The player character Altair needs to sit on a bench nearby and overhear the conversation between two NPCs in order to get clues about the situation. In one of the eavesdroppings, two assassins are talking about a man named Masun who had let the Templars into the town; he exchanges letters with someone from the fortress and the letters are carried by the basket weaver. This information immediately tells Altair that the letter is important and he needs to find the basket weaver, which will lead to the next pickpocketing activity. The span of flashbacks in this level is typically a few minutes, enough for a brief conversation. Their main function is to provide information and hints for the player.

6.1.2. Speed. In the normal state, the game operates in a scene tempo, in which players feel things happen at the same speed as in real world. Of course, if we scrutinize the time used for each action, it tends to be shorter than it would be in a real town the size of Damascus. Hence, our designation as scene tempo is relative, not absolute. Players can use the controller to speed up Altair's walking and running pace, as they would do in the real world. Ellipsis is rarely used in this level. The only ellipsis we observed takes place when the player character finishes a task and naps at the Assassin's Bureau: when he walks out of the room, there is a direct cut to the next scene showing him standing up and looking recharged. This use of ellipsis is mainly for user convenience-assassins do need rest, but the player does not need to wait. The dominant use of scene and minimal use of ellipsis are a result 
TABLE 2: Summary of spatial characteristics discussed that affect narrative and gameplay experience.

\begin{tabular}{|c|c|c|c|}
\hline $\begin{array}{l}\text { Characteristics for game } \\
\text { narrative space }\end{array}$ & $\begin{array}{l}\text { Function in game } \\
\text { narrative }\end{array}$ & Function in gameplay & $\begin{array}{l}\text { Examples cited } \\
\text { (selective) }\end{array}$ \\
\hline $\begin{array}{l}\text { Topographical } \\
\text { layout }\end{array}$ & Affect plot structure & $\begin{array}{l}\text { Help create navigational } \\
\text { pattern }\end{array}$ & Assassin's Creed BioShock \\
\hline $\begin{array}{l}\text { Topographical } \\
\text { spatial opposition }\end{array}$ & $\begin{array}{l}\text { Help group narrative } \\
\text { events; Help create } \\
\text { conventions (e.g., } \\
\text { dangerous space versus } \\
\text { safe space) to affect } \\
\text { emotional experience }\end{array}$ & $\begin{array}{l}\text { Help shape navigational } \\
\text { experience; Help create } \\
\text { conventions to affect } \\
\text { decision making }\end{array}$ & $\begin{array}{l}\text { Assassin's Creed; } \\
\text { Assassin's Creed II }\end{array}$ \\
\hline $\begin{array}{l}\text { Operational } \\
\text { character mobility } \\
\text { object mobility }\end{array}$ & $\begin{array}{l}\text { Help create the } \\
\text { storyworld; Help the plot } \\
\text { development; Affect the } \\
\text { variability of narrative }\end{array}$ & $\begin{array}{l}\text { Ease or intensify } \\
\text { gameplay; affect the } \\
\text { variability of gameplay }\end{array}$ & $\begin{array}{l}\text { Prince of Persia: Sands of } \\
\text { Time; Assassin's Creed; } \\
\text { Fable II; Heavy Rain }\end{array}$ \\
\hline $\begin{array}{l}\text { Operational } \\
\text { paths and axes }\end{array}$ & $\begin{array}{l}\text { Help shape/restrain the } \\
\text { plot structure }\end{array}$ & $\begin{array}{l}\text { Help create navigational } \\
\text { pattern; Affect the } \\
\text { degree of repetition of } \\
\text { gameplay }\end{array}$ & $\begin{array}{l}\text { Assassin's Creed; } \\
\text { Planescape Torment }\end{array}$ \\
\hline $\begin{array}{l}\text { Presentational } \\
\text { on-screen space } \\
\text { perspective }\end{array}$ & $\begin{array}{l}\text { Stylistic/genre } \\
\text { storytelling; Choice of } \\
\text { first-person versus } \\
\text { third-person help shape } \\
\text { emotional experience }\end{array}$ & $\begin{array}{l}\text { Affect the continuity of } \\
\text { game space; Affect player } \\
\text { control of view; Camera } \\
\text { as a guiding device }\end{array}$ & $\begin{array}{l}\text { God of War; Assassin's } \\
\text { Creed; Assassin's Creed II; } \\
\text { Prince of Persia: Sands of } \\
\text { Time; Max Payne }\end{array}$ \\
\hline $\begin{array}{l}\text { Presentational } \\
\text { acoustic space }\end{array}$ & $\begin{array}{l}\text { Set the mood, create } \\
\text { tension, and enhance the } \\
\text { realism. }\end{array}$ & $\begin{array}{l}\text { Provides feedback and } \\
\text { hints for players' next } \\
\text { moves; Basic game } \\
\text { mechanic (e.g., in } \\
\text { rhythm games) }\end{array}$ & $\begin{array}{l}\text { Fable II; Assassin's Creed; } \\
\text { Rock Band; Dance Dance } \\
\text { Revolution }\end{array}$ \\
\hline $\begin{array}{l}\text { Presentational } \\
\text { spatial segmentation }\end{array}$ & $\begin{array}{l}\text { Help group narrative } \\
\text { events }\end{array}$ & $\begin{array}{l}\text { Set the boundaries of } \\
\text { gameplay; help create } \\
\text { game levels }\end{array}$ & $\begin{array}{l}\text { Prince of Persia: Sands of } \\
\text { Time; Fable II; Assassin's } \\
\text { Creed }\end{array}$ \\
\hline $\begin{array}{l}\text { Presentational } \\
\text { screen interface }\end{array}$ & $\begin{array}{l}\text { Provide information; } \\
\text { stylistic/genre storytelling } \\
\text { (e.g., split screen) }\end{array}$ & $\begin{array}{l}\text { Facilitate player control } \\
\text { for both gameplay and } \\
\text { camera view; provide } \\
\text { information and } \\
\text { instructions }\end{array}$ & $\begin{array}{l}\text { Assassin's Creed; Fable II; } \\
\text { High Velocity; Heavy } \\
\text { Rain }\end{array}$ \\
\hline
\end{tabular}

of the signature fluid design of Assassin's Creed. To our knowledge, the other tempi, including summary and stretch, are absent in this level. Pause is not embedded within the storytelling but can be accessed using the player control. Players can pause in order to review the game state and game world or to put the game on standby while they do other things.

6.1.3. Frequency. Iteration is a frequency mode that is not used in this game, consistent with its absence in most games. The story events tend to be presented singularly. However, repetitions - single event being presented multiple timesare not rare in this level. The first type of repetition is typical in games; namely, repetition due to the death of the player character. In Assassin's Creed, when the player character dies due to a failed fight, the fight will be replayed so the player can repeat until succeeding. Another form of repetition is related to NPC behaviour in the game. Those NPCs who are bound to a specific location-that is, characters without mobility - tend to repeat what they do and say. Whenever the player character gets close to a "save citizen" venue, where several guards are troubling a citizen, a soundscape of their conversations will be played. If the player leaves the venue and comes back later, he hears the same conversations. This repetition will only stop after the player character saves the citizen by killing those guards. This second type of repetition reflects poor design for the nonmobile characters. This weakness is shared by many games, decreasing realism and undermining Murray's conception of "immersion" within interactive narrative. In fact, none of the level's use of repetition adds to the quality of the game's storytelling.

6.1.4. Polychrony and Narrative Variation. In the beginning of our temporal analysis, we mentioned that for the same memory block, players can select different tasks and follow different sequences during each replay. In Murray's aesthetic framework, this transformative capacity of the game's design increases a player's sense of agency [33]. Players sense the impact their choices have on the story's variations and the subsequent development of deeper meaning. 


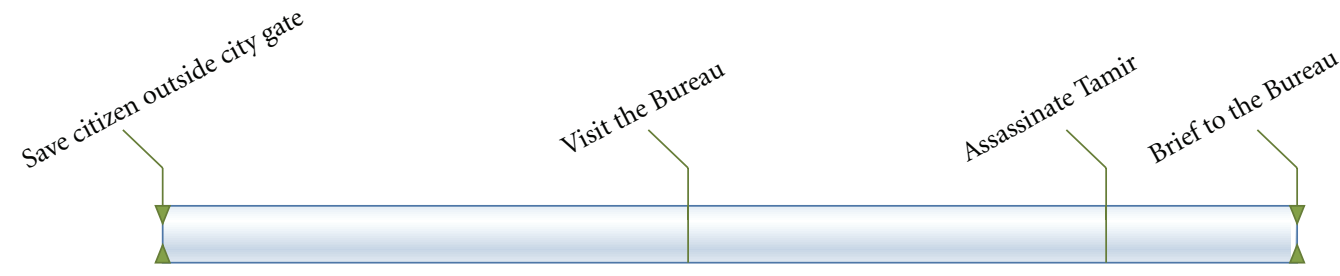

FIgURE 3: Damascus operational timeline: four mandatory events with fixed ordering.

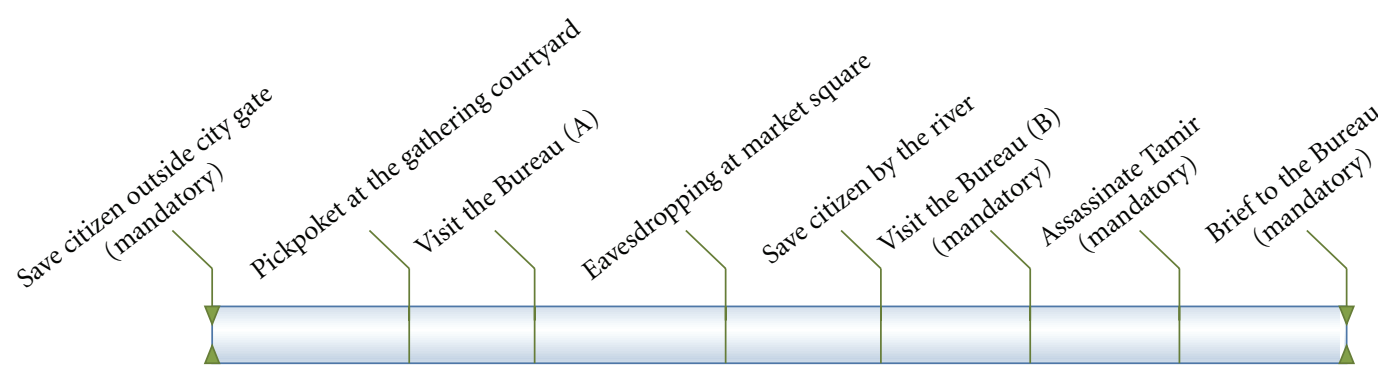

FIgURE 4: Damascus operational timeline: Sequence A.

As a general rule in Assassin's Creed, before the player visits a new subspace, that area remains concealed (i.e., black) until the player climbs to the top of a tower and "scans" the area from the vantage point. This scan, or "scaling the viewpoint," is generally the first thing to do in a space. After the scaling, the corresponding area is revealed on the map and the player can then become active in that area. In the Damascus Poor District level, the main objective is to assassinate the black market merchant Tamir. Before entering Damascus, the player character has to save a citizen close to the city gate. After the entrance, another "mandatory" task the player needs to carry out before the assassination is gather information from the leader of Damascus Assassins at the Assassin's Bureau. The player character will then be able to assassinate Tamir after finishing at least two investigation activities (pickpocketing, eavesdropping, or interrogation). After the assassination, he must return to the Bureau for a briefing in order to finish this game level. To summarize, there are only four events sequenced in a fixed order but they are not exactly positioned on the timeline. The inexact positioning is necessary to make room for player freedom. Figure 3 shows the fixed sequence of the Damascus level; other than the first and last events happening at the two ends of the timeline (marked by double triangles in the graph), events in the middle do not own a fixed time point. Figure 4 illustrates the sequence of one possible play. Aside from the beginning and end activities as well as "Assassinate Tamir," the ordering of the activities is determined by the player.

Our analysis reveals that the design of Assassin's Creed is very successful in creating variations in the narrative order. By giving the player great control over the ordering and timing of their activities, the interactivity of the game narrative is considerably enhanced. One can easily raise a question here: does the random ordering affect the narrative logic? We went back to the game, performed multiple plays and concluded that the narrative logic is not influenced by the various sequence orders. Scaling viewpoints only reveals the map whereas saving citizens only enhances the player character's reputation so that the saved citizens will provide help latter. Neither activity entails a plot function that is significant in its own right. There is some low-level narrative logic inherent in the investigation activities. Some of the dialogues deliver useful operational information regarding the assassination, such as the background information on Tamir and the venue where he will appear. But other than the practical implications, the narrative weight is low and no ordering logic is required. We did encounter, in one play (shown as Sequence B in Figure 5), a situation where the logic is affected, but we were pleased to note that the game had intelligently varied the relevant dialogue. Unlike in Sequence A, we did an eavesdropping and the pickpocketing before seeing the Bureau leader. In Sequence A (Figure 4), during the first visit, the Bureau leader reveals some information on Tamir and suggests a number of locations in the Souk area where Altiar can begin his investigation; during the second visit, he praises Altair's capability and gives permission (i.e., gives the feather marker) to Altair to assassinate Tamir. In Sequence B, since both investigations have already revealed much information about Tamir, the conversation combines the two dialogues in Sequence $\mathrm{A}$ and alters slightly to accommodate what Altair has done in this sequence.

\subsection{Spatial Analysis}

6.2.1. Topographical View. Taking the Damascus Poor District as a static space, we see this area as an example of any medieval middle-east city district. In fact, Assassin's Creed's game spaces were modelled after the geography of the actual middle-east cities (around the Holy Land) in 12th century. The architectural design and visual details are based on 


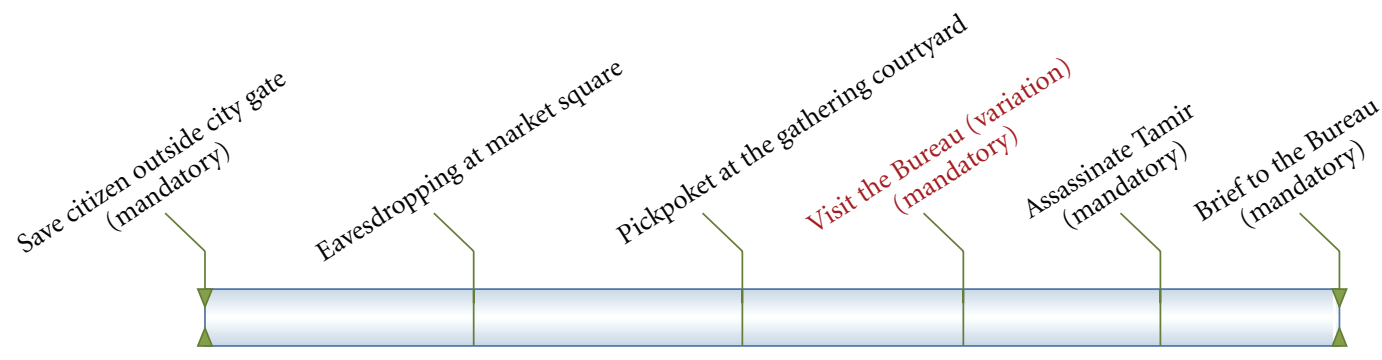

FIgUre 5: Damascus operational timeline: Sequence B.

historical documents and thus look very realistic [50]. The city is guarded with walls and gates. Within the city are the mosques, markets, alleys, and various types of courtyards. Like any populated city, locations are very well connected, which means that more than one path can lead one from one location to another. Thus we can consider the space to be a network layout, with a clear boundary for the district and an evenly distributed density across the area. Figure 6 selectively shows six landmarks that have visually distinct features to make them stand out. Since the real setting of this district is quite dense, we use a limited number of symbolic paths just to show a high-level view of the layout.

As previously mentioned, the game features an architectural style where rooftops of large structures are often connected, and alleys between structures in this poor district tend to be narrow. This two-level vertical structure offers the possibility for the player to take shortcuts by jumping around between roofs, which makes parkour one of the game's most distinct gameplay features. The term parkour is originated from a French physical discipline where participants overcome obstacles and run along a route. The word is used in games nowadays with the general meaning of free running. In general, the security level on the rooftop is low, and the guards on the ground cannot see activities on the rooftop; the rooftop thus facilitates stealth in the gameplay. The twolevel vertical design, as well as the network layout, directly influences the space's operational structure by shaping the navigation pattern: the former gives players control of the pacing and the latter creates multiple options for their navigation routes.

6.2.2. Operational View. In the Damascus Poor District, the protagonist needs to complete 2 out of 6 main tasks from Memory Strands 1 to 6 . He will then be able to report back to the Bureau (Memory Strand 7) and finally assassinate Tamir (Memory Strand 8), before this level is finished. There are also two types of side tasks in the game operation. One is to scale the viewpoints; the other is to save citizens who are in trouble. Figure 7 illustrates the main tasks based on their locations on the map. These tasks take place in a fixed location, which also implies the immobility of most of the NPCs; that is, characters are bound to one location or to a very limited range. The only exception is the vigilantes, who seem to appear wherever the protagonist is in trouble, providing that he is in good credit (gained by saving citizens).
From the diagram we can see that there is really no axis of centralized actions or happenings. Task locations are evenly scattered around, which means that the player is encouraged to navigate through the entire space.

The immobility of characters and associated actions could potentially make the game dull and lead to poor replayability because after the first time visiting a place, the player will see exactly the same characters and actions during a revisit until the characters are killed or the associated actions are taken, as we have discussed earlier in temporal analysis. However, the large and richly designed space makes up for this weakness in several ways. First, by performing a more-than-required number of tasks, the player can choose to do different things or visit different places for each play. Second, the rich and beautifully rendered environment entices the player to explore and discover new scenery. Third, the network layout provides a number of paths between any two locations, which makes the navigation more interesting and less repetitive.

The provision of a large and well-designed space with full navigational freedom to the player does compensate for the immobility of so many of the characters. However, it also induces another potential problem. With so many options for what to do and where to go, the player can become uncertain about what is the goal for this particular level and what to do next, or how to finish the experience and get out of this town. To make things worse, the game lacks axes in its operational space. The cure for this problem is twofold: one is posting real-time, on-screen instructions and task-indicating marks on the in-game map; the other is creating side tasks that indirectly help unfold the game plot. Scaling viewpoints is such a side task that especially addresses player uncertainty. When the player scales a viewpoint for the first time (by climbing to the top of tower and getting a panoramic bird's eye view), the surrounding area on the in-game city map, originally all dark, will be revealed. This can be related to a game design concept called "fog of war," where the focus area is revealed but other areas on the map are covered; examples can be found in strategy game Civilization and role-playing game World of Warcraft. When all viewpoints are scaled, the whole Damascus map will be shown clearly on the city map (which can be called up on demand). The viewpointscaling endeavour helps players, self-orient by giving a sense of which area has been visited and which has not. With the viewpoints being evenly spread out (see Figure 6), the player 


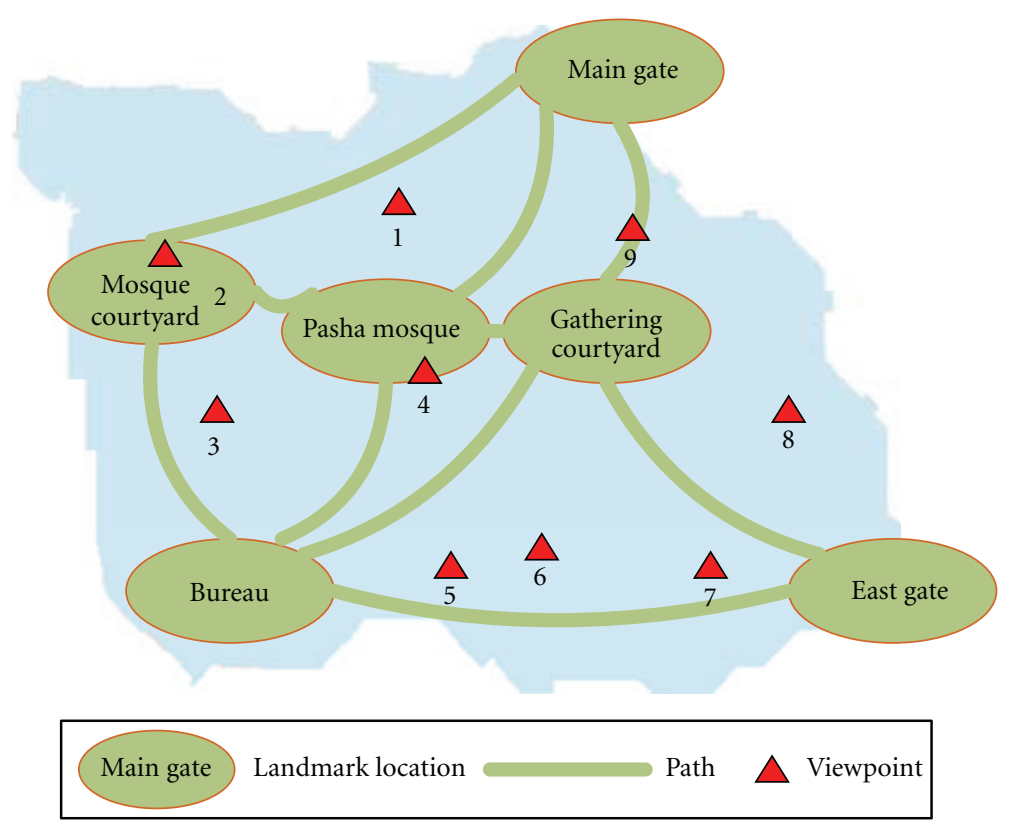

FIgURe 6: Damascus Poor District: topographical view.

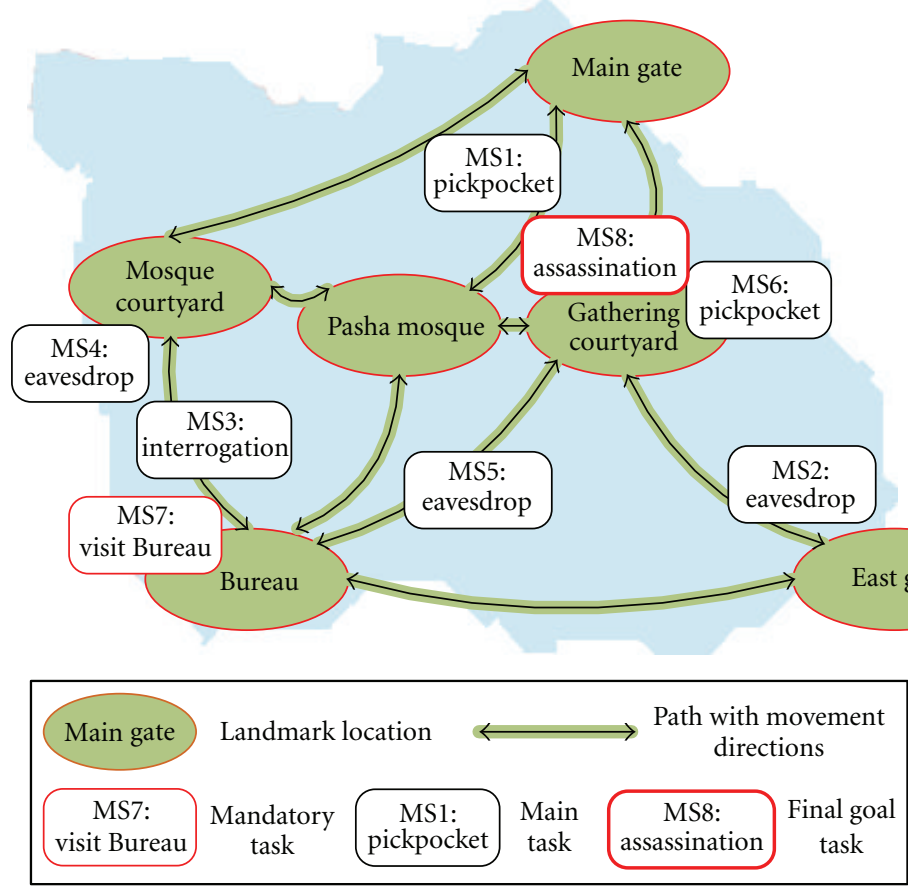

Figure 7: Damascus Poor District: operational view.

is prevented from getting lost in a labyrinth-like space and from neglecting a new area that potentially has a mandatory task to do.

6.2.3. Presentational View. Assassin's Creed is very cinematic in its visual style. There is no surprise that we see a lot of cinematic techniques in use here. Having a thirdperson physical perspective, the player has full control of the camera angles, plus an "eagle vision" that highlights NPCs of interest. On the other hand, the player assumes a first-person psychological perspective for Altair the Assassin most of the time. The off-screen space and on-screen space together form a 3D story world.

The organization of the game plot is based on locations. Generally speaking, each town is a game level that has its own action style and spatial structure. Within Damascus Poor District, however, there is no visible segmentation. The space is continuous, which results in a fluid representation. 
The camera follows the protagonist traversing the space and presents the space accordingly. The fluidity is stressed so much that even in some of the cut-scenes (e.g., at the Bureau), the player can keep adjusting his camera view and walking about, while participating in a prescripted conversation. This seamless interaction between the player avatar and NPCs reduces some of the perceived disadvantages of cutscenes (e.g., the player getting bored due to loss of control). This is important-every task scene is interspersed with cutscenes to carry on conversations.

The sound design of Assassin's Creed is outstanding. The ambient music creates a mood evocative of the ancient middle-eastern culture. As part of the interactive environment, the soundtrack reacts to what the player does in real time. If the player avatar kills a guard, the NPCs around him will scream and shout out their feelings about him. When the player character is exposed to the city guards, an ambient alert alarm will sound; in the meanwhile, the accompanied background music will change into faster rhythm when the player character is chased by the guards. This dynamic design effectively intensifies the player's narrative, emotional, and gameplay experiences.

The screen layout of this game uses overlays with an alternative interface screen showing the city map (e.g., Figure 2). The play screen presents the game space. Game instructions and hints are overlaid semitransparently on the corners of the screen (Figure 8). At the bottom right corner of the screen lies the mini GPS indicating the direction and distance to the target. In the alternative map screen, players can set a marker on the map interface (Figure 2), which in turn will show up in the GPS on the play screen. Assassin's Creed's interface design creates an interesting, somewhat explicit link between the three levels of the space. It provides players a topographical view in the form of a town map. The map screen has marks for the locations of tasks, providing an overall operational view of the space and hints about appropriate movement and navigation. Not all games have these explicit links between the three views of their narrative space; nevertheless, these links always exist, converging design efforts at all three levels: topographical, operational, and presentational.

6.3. Plot Structure. The interactive narrative structure of Assassin's Creed is a typical foldback structure. The plot consists of seven memory blocks. The structure of all the memory blocks is almost identical, except for the first memory block that can be seen as the training block. The sequencing of the seven blocks has no particular temporal design. Within each block, there are two to three assassination missions to complete. These missions are the actual game levels and can also be regarded as plot units. The structure of each mission is again identical. Within a block, players can complete the missions in any order. As we can see, this repetitive structural design and random order of bigger events on the high level does not help build a strong dramatic tension arc-the tension level of each mission or block is equal. However, within each mission, although the sequencing mechanism gives players certain freedom, we can see an arc in the general flow of the plot (see Figure 9). The investigation tasks can be seen as the exposition or setup act where the clues about the assassination target are given or discovered. In the second act, the player character finds the target and kills him, which pushes this unit of plot to the climax. In the third act, the player character makes his escape and the mission is completed.

In Assassin's Creed, all missions are bound to a location. Hence, rather than sequencing based on the three-act structure, at a high level the plot is structured based on location. Within a mission, the mandatory investigation tasks are also location-bound. Since the order of investigation tasks is random, the player needs to have a good grasp on the topographical layout of the town in order to engage in efficient play. In this way, the temporal design in effect is woven into the location design and played out in the enactment of plot and gameplay.

\section{Conclusion}

The study of game time and space has seen many interdisciplinary approaches drawing on a variety of traditions and methodologies. However, there is rarely a systematic discussion of how time and space are structured in game narratives and more specifically, what the key devices and characteristics are involved in the structuring process. We therefore decided to adapt and apply mature frameworks drawn from broader narrative theory in the analysis of story design and experience in games. Through investigations in fields of both narrative analysis and game studies and design, we develop a descriptive framework that organizes important time- and space-related issues into categories and designates characteristics to each category. Our framework for game time consists of the classic narratological categorization of order, speed, frequency, as well as polychrony, a crucial notion originally proposed by Herman addressing fuzzy temporality, which serves as the key to narrative interactivity. Based on these categories, we account temporal devices that support narrative and gameplay. Our framework for game narrative space, inspired mainly by Zoran and Herman's works, consists of three views that show the topographical, operational, and presentational structure of the narrative space in games. Within this framework, we lay out some important characteristics that define the qualities of the space. Through the crosschecking of game examples and a case analysis, our game-specific framework is found to be able to support the understanding of game narrative design.

A survey of the field of game studies encounters several important frameworks that address either time or space in games. Among these frameworks, Nitsche's is one that explicitly adapts narratology in the discussion of the structure of game space in one of the three parts forming his whole book (i.e., structure, presentation, functionality). Nitsche's discussion focuses on the meaning of the story in games and how to understand and adapt the fundamental concepts of story, plot and discourse in the context of game. However, this high-level conceptual discussion leaves the detailed methods for analysis and design of game narrative space largely unaddressed. Moreover, Nitsche's book interconnects the conception of five principle spaces: rule-based space, 


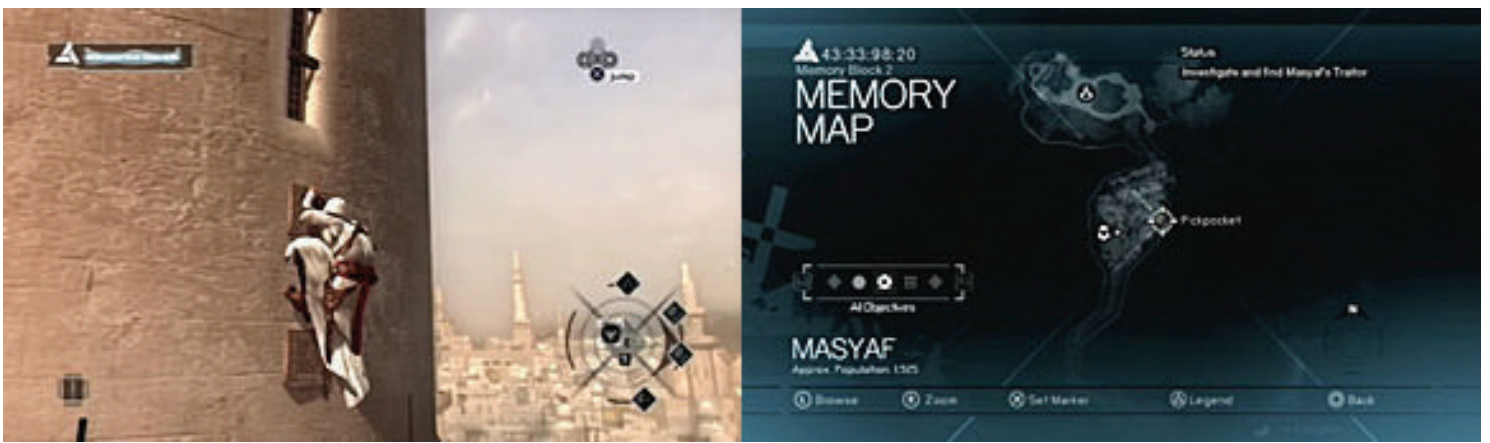

FIGURE 8: The play screen and map menu of Assassin's Creed (source: Ubisoft, 2007; image from IGN.com, used with permission).

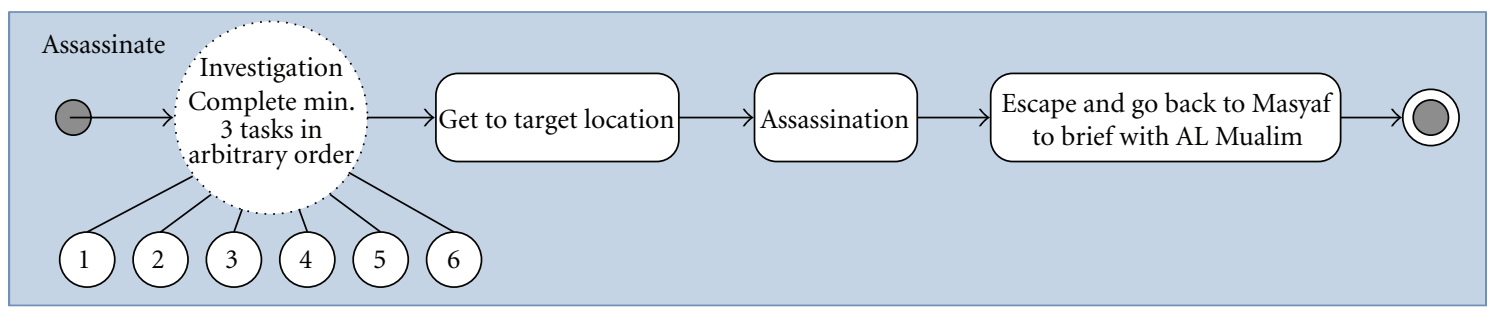

Figure 9: A plot unit in Assassin's Creed.

mediated space, fictional space, play space, and social space. Although this integrated view on game space is of great value, it is hard to find principles specific to narrative design. Jenkin's seminal article on narrative spaces gives us a good starting point to examine the relation between narrative and space, but it contains no specific insights on the structure of time and space. Other frameworks we investigated have even less focus on game storytelling. In Section 2, we introduced several frameworks for game time. These frameworks, built on multiple time schemes or time frames, cover some important concepts of game temporality, though not specifically for narrative comprehension. In addition, in our exploration, we identified the key insight that making narrative interactive lies in the fuzzy temporality that helps create plot variations and thus breaks the linearity of the story. This issue is not addressed in clear terms by any game scholars we have surveyed, although Ryan addresses plot variation in her studies of interactive narrative in terms different from ours [51]. Other frameworks for game space are less comprehensive than Nitsche's and not focused on storytelling, either. For instance, McGregor summarizes six patterns of use of space in games, which is a good descriptive framework to study the relation between space and gameplay [52].

In games, time and space work together to anchor textual, visual, auditory, and other interactive cues in the digital game, from which game players can build their mental story world. Players' narrative experiences are greatly influenced by the ways these cues are embedded in games to form temporal and spatial patterns. Devices and characteristics that facilitate both storytelling and gameplay are especially important in the creation of engaging plot and play. These are the vehicles for conjoining interactive experience and narrative experience within an integrated dynamic. Treating games as interactive narrative texts, this paper adapts narrative theory in the explication of the structural aspects of time and space in game storytelling. These insights are instantiated within a conceptual framework that incorporates the key factors in the design of game narrative. This framework can be usefully applied in the study of specific games, and can also serve as a basis for critical analysis on a larger scale, providing a methodology for comparing narrative design across different games. As the boundary between digital games and interactive storytelling systems is not clear cut and they share many principles discussed in our work, we believe that our framework can effectively be extended into the domain of interactive storytelling.

\section{References}

[1] M. J. P. Wolf, "Narrative in the video game," in The Medium of the Video Game, M. J. P. Wolf, Ed., University of Texas Press, Austin, Tex, USA, 2001.

[2] T. Bridgeman, "Time and space," in The Cambridge Companion to Narrative, D. Herman, Ed., pp. 52-65, Cambridge University Press, Cambridge, Mass, USA, 2007.

[3] E. Aarseth, "Allegories of space: the question of spatiality in computer games," in Space Time Play: Computer Games, Architecture and Urbanism: The Next Level, F. von Borries, S. P. Walz, and M. Böttger, Eds., pp. 44-47, Birkhäuser, Berlin, Germany, 2007.

[4] M. J. P. Wolf, "Space in the video game," in The Medium of Video Game, J. P. M. Wolf, Ed., pp. 51-75, University of Texas Press, Austin, Tex, USA, 2001.

[5] D. J. Boron, "A short history of digital gamespace," in Space Time Play: Computer Games, Architecture and Urbanism: The Next Level, F. von Borries, S. P. Walz, and M. Böttger, Eds., pp. 26-31, Birkhäuser, Berlin, Germany, 2007. 
[6] H. Jenkins, "Game design as narrative architecture," in First Person: New Media as Story, Performance, and Game, N. Wardrip-Fruin and P. Harrigan, Eds., pp. 118-130, MIT Press, Cambridge, Mass, USA, 2004.

[7] M. Nitsche, "Mapping time in video games," in Situated Play, Proceedings of DiGRA 2007 Conference, pp. 145-151, 2007.

[8] M. LeBlanc, "Tools for creating dramatic game dynamics," in The Game Design Reader: A Rules of Play Anthology, K. Salen and E. Zimmerman, Eds., pp. 438-459, MIT Press, Cambridge, Mass, USA, 2006.

[9] D. Arsenault and B. Perron, "In the frame of the magic cycle: the circle(s) of gameplay," in The Video Game Theory Reader 2, B. Perron and M. J. P. Wolf, Eds., pp. 109-131, Routledge, New York, NY, USA, 2009.

[10] J. Juul, Half-Real: Video Games between Real Rules and Fictional Worlds, MIT Press, Cambridge, Mass, USA, 2005.

[11] J. P. Zagal and M. Mateas, "Temporal frames: a unifying framework for the analysis of game temporality," in Situated Play, Proceedings of DiGRA 2007 Conference, pp. 516-522, 2007.

[12] M. Ryan, "Beyond myth and metaphor-the case of narrative in digital media," Game Studies: The International Journal of Computer Game Research, vol. 1, no. 1, 2001, http://www.gamestudies.org/0101/ryan/ .

[13] M. M. Bakhtin, "Forms of time and of the chronotope in the novel: notes toward a historical poetics," in The Dialogic Imagination: Four Essays, M. M. Bakhtin and M. Holquist, Eds., pp. 84-258, Univeristy of Texas Press, Austin, Tex, USA, 1981.

[14] G. Zoran, "Towards a theory of space in narrative," Poetics Today, vol. 5, pp. 309-335, 1984.

[15] D. Herman, Story Logic: Problems and Possibilities of Narrative, University of Nebraska Press, Lincoln, Neb, USA, 2002.

[16] M. Nitsche, Video Game Spaces: Image, Play, and Structure in 3D Game Worlds, MIT Press, Cambridge, Mass, USA, 2008.

[17] G. Genette, Narrative Discourse: An Essay in Method, Cornell University Press, Ithaca, NY, USA, 1980.

[18] M. Eskelinen, "Towards computer game studies," in First Person: New Media as Story, Performance and Game, N. Wardrip-Fruin and P. Harrigan, Eds., pp. 36-44, MIT Press, Cambrige, Mass, USA, 2004.

[19] J. Juul, "Introduction to game time," in First Person: New Media as Story, Performance, and Game, N. Wardrip-Fruin and P. Harrigan, Eds., pp. 131-142, MIT Press, Cambridge, Mass, USA, 2004.

[20] M. Hitchens, "Time and computer games or "no, that's not what happened," in Proceedings of the 3rd Australasian Conference on Interactive Entertainment, pp. 44-51, 2006.

[21] A. Tychsen, M. Hitchens, and A. Drachen, "Game time: modeling and analyzing time in multiplayer and massively multiplayer games," Games and Culture, vol. 4, no. 2, pp. 170201, 2009.

[22] M. Bal, Narratology: Introduction to the Theory of Narrative, University of Toronto Press, Toronto, Canada, 3rd edition, 2009.

[23] G. Prince, Dictionary of Narratology, University of Nebraska Press, Lincoln, Neb, USA, 2nd edition, 2003.

[24] S. Chatman, Story and Discourse: Narrative Structure in Fiction and Film, Cornell University Press, Ithaca, NY, USA, 1978.

[25] E. Adams, Fundamentals of Game Design, New Riders, Berkeley, Calif, USA, 2nd edition, 2010.

[26] J. E. Brand, S. Knight, and J. Majewski, "The diverse worlds of computer games: a content analysis of spaces, populations, styles and narratives," in Proceedings of the Digital Games
Research Association Conference (DIGRA '03), Utrecht, The Netherlands, November 2003.

[27] L. Soulban and H. Orkin, "Writing for first-person shooters," in Writing for Video Game Genres: From FPS to RPG, W. Despain, Ed., pp. 51-68, A K Peters, Wellesley, Mass, USA, 2009.

[28] J. Mechner, "The sands of time: crafting a video game story," in Second Person: Role-Playing and Story in Games and Playable Media, P. Harrigan and N. Wardrip-Fruin, Eds., pp. 111-120, MIT Press, Cambridge, Mass, USA, 2007.

[29] H. Kelly, "Animal crossing: a game in time," in Space Time Play: Computer Games, Architecture and Urbanism: The Next Level, F. von Borries, S. P. Walz, and M. Böttger, Eds., pp. 180-181, Birkhäuser, Berlin, Germany, 2007.

[30] P. Ruffino, "Max payne: the dream of control over time," in Space Time Play: Computer Games, Architecture and Urbanism: The Next Level, F. von Borries, S. P. Walz, and M. Böttger, Eds., pp. 70-71, Birkhäuser, Berlin, Germany, 2007.

[31] M. Kinder, "The subversive potential of the pseudo-iterative," Film Quarterly, vol. 43, pp. 2-16, 1989.

[32] N. Montfort, "Ordering events in interactive fiction narratives," in Proceedings of the AAAI Fall Symposium on Intelligent Narrative Technologies, pp. 87-94, 2007.

[33] J. H. Murray, Hamlet on the Holodeck: The Future of Narrative in Cyberspace, Free Press, New York, NY, USA, 1997.

[34] S. Gaynor, Reorienteering: spatial organization in BioShock, 2009, http://fullbright.blogspot.com/2009/04/reorienteeringspatial-organization-in.html .

[35] L. Manovich, The Language of New Media, MIT Press, Cambridge, Mass, USA, 2001.

[36] D. Carr, "Space, navigation and affect," in Computer Games: Text, Narrative and Play, D. Carr, D. Buckingham, A. Burn, and G. Schott, Eds., pp. 59-71, Polity Press, Cambridge, UK, 2006.

[37] J. Carlquist, "Playing the story: computer games as a narrative genre," Human IT, vol. 6, no. 3, pp. 7-53, 2002.

[38] M. Grimshaw and G. Schott, "Situating gaming as a sonic experience: the acoustic ecology of first-person shooters," in Situated Play: Proceedings of the Digital Games Research Association Conference, pp. 474-481, 2007.

[39] J. P. Zagal, C. Fernández-Vara, and M. Mateas, "Rounds, levels, and waves: the early evolution of gameplay segmentation," Games and Culture, vol. 3, no. 2, pp. 175-198, 2008.

[40] M. S. Meadows, Pause \& Effect: The Art of Interactive Narrative, New Riders, Indianapolis, Ind, USA, 2003.

[41] R. Rouse III, Game Design Theory and Practice, 2nd edition, 2005.

[42] D. Bordwell, Poetics of Cinema, Routledge, New York, NY, USA, 2008.

[43] M. Mateas and A. Stern, "Structuring content in the façade interactive drama architecture," in Proceedings of the 1st Artificial Intelligence and Interactive Digital Entertainment Conference (AIIDE '05), pp. 93-99, June 2005.

[44] L. M. Barros and S. R. Musse, "Towards consistency in interactive storytelling: tension arcs and dead-ends," Computers in Entertainment, vol. 6, no. 3, article 43, pp. 1-17, 2008.

[45] D. Thue, V. Bulitko, M. Spetch, and E. Wasylishen, "Interactive storytelling: a player modelling approach," in Proceedings of the 3rd Artificial Intelligence for Interactive Digital Entertainment Conference (AIIDE'07), pp. 43-48, Stanford, Calif, USA, 2007.

[46] H. Jenkins, "Narrative spaces," in Space Time Play: Computer Games, Architecture and Urbanism: The Next Level, F. von Borries, S. P. Walz, and M. Böttger, Eds., pp. 56-60, Birkhäuser, Berlin, Germany, 2007. 
[47] M. J. P. Wolf, "Time in the video game," in The Medium of Video Game, J. P. M. Wolf, Ed., pp. 77-91, University of Texas Press, Austin, Tex, USA, 2001.

[48] B. Mallon and B. Webb, "Stand up and take your place: identifying narrative elements in narrative adventure and roleplay games," Computer in Entertainment, vol. 3, no. 1, pp. 119, 2005.

[49] J. P. Zagal, M. Mateas, C. Fernández-Vara, B. Hochhalter, and N. Lichti, "Towards an ontological language for game analysis," in Proceedings of the DiGRA Conference: Changing Views-Worlds in Pla (DiGRA '05), 2005.

[50] M. Seif El-Nasr, M. Al-Saati, S. Niedenthal, and D. Milam, "Assassin's creed: a multi-cultural read," Loading, vol. 2, no. 3, 2008.

[51] M. Ryan, Avatars of Story, University of Minnesota Press, Minneapolis, Minn, USA, 2006.

[52] G. L. McGregor, "Situations of play: patterns of spatial use in videogames," in Situated Play: Proceedings of the Digital Games Research Association Conference, pp. 537-545, 2007. 

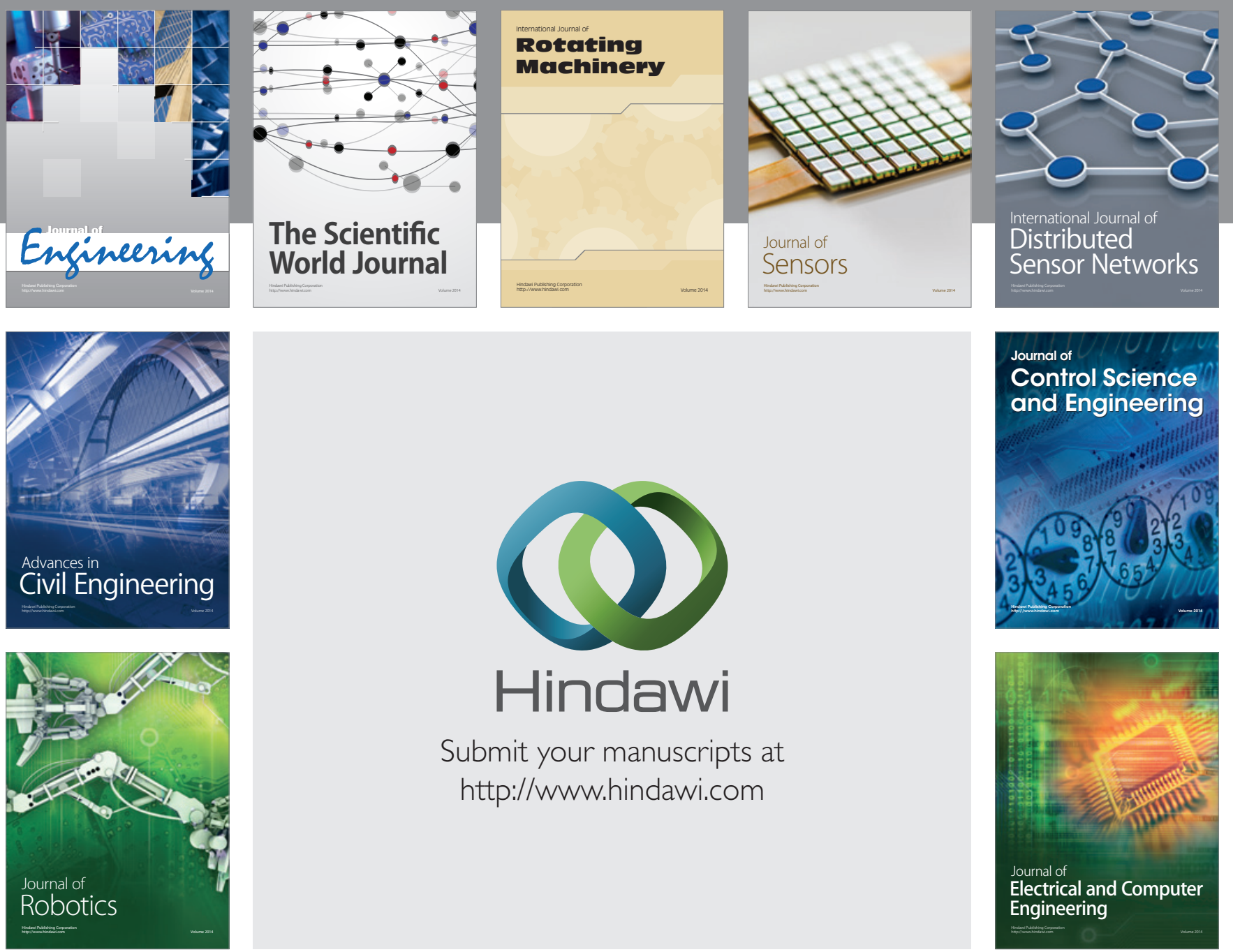

Submit your manuscripts at

http://www.hindawi.com
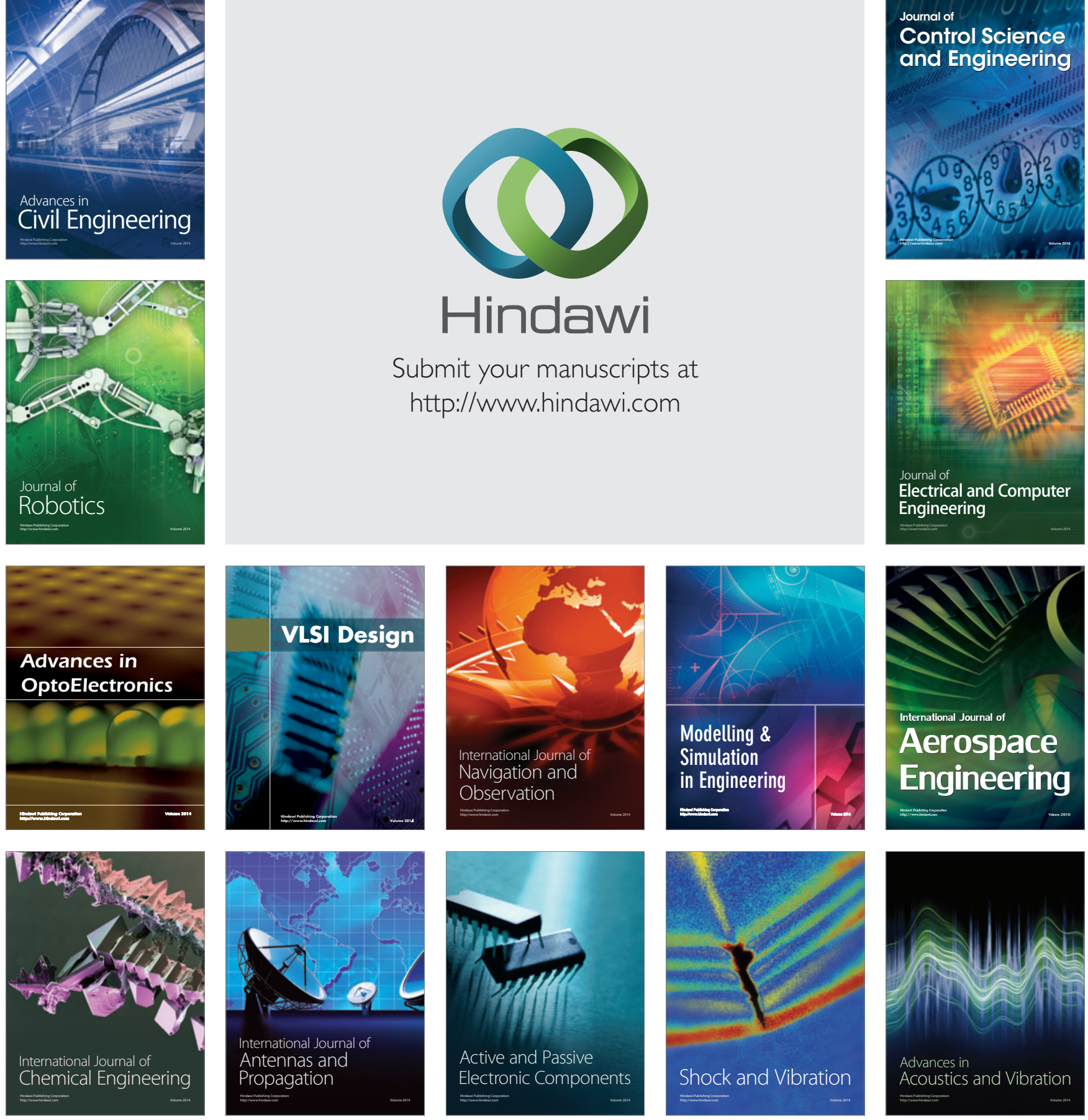